\title{
On "Hyperboloidal" Cauchy Data for Vacuum Einstein Equations and Obstructions to Smoothness of Scri
}

\author{
Lars Andersson ${ }^{1}$, Piotr T. Chruściel ${ }^{2, \star}$ \\ 1 Department of Mathematics, Royal Institute of Technology, S-10044 Stockholm, Sweden. \\ e-mail: larsa@math.kth.se \\ 2 Centre for Mathematics and its Applications, Australian National University, \\ G.P.O. Box 4, Canberra A.C.T. 2601, Australia. \\ e-mail: pxc851@huxley.anu.edu.au
}

Received: 1 April 1993

\begin{abstract}
The relationship between the geometric properties of "hyperboloidal" Cauchy data for vacuum Einstein equations at the conformal boundary of the initial data surface and between the space-time geometry is analyzed in detail. We prove that a necessary condition for existence of a smooth or a polyhomogeneous Scri (i.e., a Scri around which the metric is expandable in terms of $r^{-j} \log ^{i} r$ rather than in terms of $r^{-\jmath}$ ) is the vanishing of the shear of the conformal boundary of the initial data surface. We derive the "boundary constraints" which have to be satisfied by an initial data set for compatibility with Friedrich's conformal framework. We show that a sufficient condition for existence of a smooth Scri (not necessarily complete) is the vanishing of the shear of the conformal boundary of the initial data surface and smoothness up to boundary of the conformally rescaled initial data. We also show that the occurrence of some log terms in an asymptotic expansion at the conformal boundary of solutions of the constraint equations is related to the non-vanishing of the Weyl tensor at the conformal boundary.
\end{abstract}

\section{Introduction}

In the papers of Bondi et al. [3], Sachs [18] and Penrose [17] which laid the foundations of our present understanding of the gravitational radiation, some rather strong hypotheses about the asymptotic behaviour of the gravitational field in lightlike directions were postulated. In a recent study by ourselves [1] (cf. also [2]) of the asymptotic properties of solutions of constraint equations on spacelike hypersurfaces intersecting "Scri" transversally it has, however, been observed that generic Cauchy data constructed in such a setting by the "conformal method" failed to be smoothly

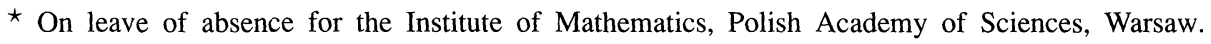
Supported in part by KBN grant \#2 10479101 
extendable, after appropriate rescalings, to the conformal boundary ${ }^{1}$ due to the occurrence of some logarithmic terms in an asymptotic expansion of the fields near the conformal boundary. This behaviour was observed even though the "seed fields" used in the conformal method were smoothly extendable to the conformal boundary. The aim of this paper is to analyze in detail the obstructions to smoothness of the solutions of the constraint equation constructed as in [1]. In order to present our results it is useful to define two tensor fields $\sigma^{ \pm}$defined on the connected component under consideration $\partial M_{i}$ of the conformal boundary $\partial M$ of the initial data surface:

$$
\sigma_{A B}^{ \pm} \equiv\left(\lambda_{A B}-\frac{h^{C D} \lambda_{C D}}{2} h_{A B}\right) \pm\left.\left(K_{A B}-\frac{h^{C D} K_{C D}}{2} h_{A B}\right)\right|_{\partial M_{\imath}}
$$

which we shall call the shear tensor of $\partial M_{i}$, where $h_{A B}$ is the induced metric on $\partial M_{i}, K_{A B}$ is (the restriction to $\partial M$ of) the extrinsic curvature $\left(K_{i j}\right)$ of $M$ in the unphysical space-time $(V, \gamma)$, and $\lambda_{A B}$ is the extrinsic curvature of $\partial M_{i}$ in $(M, g)$. In the case of Cauchy data constructed as described in Sect. 2, our results linking the geometry of the boundary of the initial data surface with the geometry of the resulting space-time can be summarized as follows:

1. Suppose that neither $\sigma^{+}$nor $\sigma^{-}$vanishes. Then there exists no development of the initial data with a smooth or polyhomogneous Scri.

2. Suppose that $\sigma^{+} \equiv 0$ or $\sigma^{-} \equiv 0$; changing the time orientation if necessary we may without loss of generality assume that $\sigma^{+} \equiv 0$ :

(a) Suppose moreover that $\left.K_{1 A}^{\log }\right|_{\partial M_{i}} \not \equiv 0$, where $K_{1 A}^{\log }$ denotes "the logarithmic part" of $K_{1 A}$, cf. Sect. 3 for details. If there exists a development with a polyhomogeneous Scri, then it is essentially polyhomogeneous, i.e., no development with a smooth Scri exists.

(b) Suppose instead that $\left.K_{1 A}^{\log }\right|_{\partial M_{\imath}} \equiv 0$.

i) Suppose moreover that $\left.K_{11}^{\mathrm{log}}\right|_{\partial M_{i}} \not \equiv 0$. If there exists a polyhomogeneous Scri, we cannot exclude the possibility that it is inessentially so, i.e., that it can be made smooth by an appropriate change of gauges.

ii) Suppose finally that we moreover have $\left.K_{11}^{\log }\right|_{\partial M_{i}} \equiv 0$. Then there exists a development which admits a smooth conformal boundary.

It should be stressed that the results linking the log terms with the non-vanishing of the Weyl tensor proved here show that the occurrence of shear and of at least some of the log terms in asymptotic expansions of physical fields at Scri is not an artefact of a bad gauge choice, including the choice of a conformal factor, or of a pathological choice of the initial data hypersurface (within the class of uniformly bounded from above and uniformly bounded away from zero, locally $C^{2}$, conformal factors and $C^{1}$ deformations of the initial data hypersurface which fix $\partial M$ ): if Scri is not shear-free (by which we mean that Eq. (3.11) does not hold), then no conformal transformation will make it shear free. Similarly if the Weyl tensor does not vanish at $\partial M$, then no gauge change in the above sense will make it vanish (cf. Sect. 3 for a more detailed discussion).

\footnotetext{
1 It has similarly been observed by Christodoulou and Klainerman [6] that generic space-times constructed by evolving "small" Cauchy data asymptotically flat in spacelike directions seem to display asymptotic behaviour incompatible with the Bondi-Sachs-Penrose (BSP) conditions (and this result has apparently been recently rigorously established: D. Christodoulou, private communications). Since the Christodoulou-Klainerman theorem is proved under quite different conditions its conclusions are, however, somewhat different from the ones obtained here
} 
Let us mention that results somewhat similar to those obtained here have been recently derived in a Bondi-Sachs type setting in [7].

The overall picture that emerges from the results of $[6,1,7]$ and from the results here is that the usual hypotheses of smoothness of Scri are overly restrictive. These results seem to indicate that the correct setup for an analysis of the gravitational radiation is that of polyhomogeneous rather than smooth functions on the conformally completed manifold, i.e., functions that have asymptotic expansions in terms of powers of $x$ and $\log x$ rather than of $x$ only. It should, however, be stressed that even though the fact that the physical fields $(\tilde{g}, \tilde{K})$ satisfy the constraint equations guarantees the existence of some vacuum development $(V, \tilde{\gamma})$, it is by no means obvious that in the case, say $\sigma^{+} \equiv 0$, the existence of some kind of compactification of $(M, \tilde{g}, \tilde{K})$ implies the existence of some useful conformal completion of $(V, \tilde{\gamma})$. The question of existence of conformal completions of $(V, \tilde{\gamma})$ with three dimensional boundaries is the most important unsolved problem of the present theory.

This paper is organized as follows: In Sect. 2 we review the results of [1] about existence and structure of solutions of constraint equations satisfying "rough" hyperboloidal conditions ${ }^{2}$. In Sect. 3 we study the relationship between the Weyl tensor at the conformal boundary and some of the log terms in the Cauchy data, cf. Theorem 3.1. We should stress that in that section we do not assume that the data have been constructed by the methods of [1], rather we study a quite general class of initial data satisfying boundary conditions, which are inspired by those that would be satisfied by data constructed in [1]. Nevertheless the class of data considered is significantly larger than that described in Sect. 2, and we have actually tried to tailor the boundary conditions to be the weakest possible for our analysis to go through without some significant difficulties. The argument in Sect. 3 is essentially a careful reexamination of the argument presented by Geroch in [12] assuming a smooth Scri. In that section we also derive some results which are necessary for further applications, cf. Theorem 3.2. It seemed of interest to rederive the results of Sect. 3 in a more direct manner, using directly the constraint equations: this is done in Sect. 4 . In that section we show that the rather indirect four-dimensional methods of Sect. 3 can be replaced by an analysis which is conceptually more straightforward but much more tedious from a calculational point of view, using $3+1$ and $2+1+1$ decompositions of the fields. In Sect. 5 we address the question of existence of a time evolution of the initial data which possesses a polyhomogeneous or smooth Scri. Theorem 5.1 is our main existence result, while Proposition 5.1 is our main non-existence result. Various conventions and definitions are given in Appendices A and E. In Appendix B we show that initial data with a "shear-free" conformal boundary can be embedded in a space-time which is asymptotically Minkowskian in light-lie directions. In Appendix $\mathrm{C}$ the notion of the extrinsic curvature of a null hypersurface is shortly discussed. Some calculational arguments needed in Sect. 3 can be found in Appendix D.

\section{The Conformal Method}

In this section we shall outline the Lichnerowicz-Choquet-Bruhat-York method for constructing solutions of the constraint equations (2.1)-(2.2) of general relativity, with

\footnotetext{
2 We use the qualification "rough" for the boundary conditions of [1] to differentiate them from those that are needed in Friedrich's evolution theorems. It is precisely the object of this paper to demonstrate with what conditions should the "rough" boundary conditions of [1] be supplemented to obtain data suitable for Friedrich's theorems
} 
asymptotic conditions appropriate to the "hyperboloidal" initial value problem $[8,9]$, cf. also $[2,1]$. Let $\bar{M}$ be a smooth, connected, Hausdorff, compact three dimensional manifold with boundary, set $M=\operatorname{int} \bar{M}, \partial \bar{M}=\partial M \equiv \bar{M} \backslash M$. Given "seed fields" $\left(g_{i j}, A^{i j}\right)$, where $g_{i j}$ is any smooth Riemannian metric on $M$ extending smoothly to $\partial M$, and $A^{i j}$ is any symmetric, traceless tensor field on $M$ extending smoothly to $\partial M$, a solution $(\tilde{g}, \tilde{K})$ of the general relativistic constraint equations

$$
\begin{gathered}
R(\tilde{g})=\tilde{g}_{i j} \tilde{g}_{k l} \tilde{K}^{i k} \tilde{K}^{j l}-\left(\tilde{g}_{i j} \tilde{K}^{i j}\right)^{2}, \\
\tilde{D}_{i}\left(\tilde{K}^{i j}-\tilde{g}_{k l} \tilde{K}^{k l} \tilde{g}^{i j}\right)=0,
\end{gathered}
$$

where $\tilde{D}_{i}$ is the Levi-Civita connection of $\tilde{g}_{i j}$, and $R(f)$ is the Ricci scalar of a metric $f$, satisfying the condition

$$
\tilde{K} \equiv \tilde{g}_{i j} \tilde{K}^{i j}=3 \varepsilon, \quad \varepsilon= \pm 1
$$

can be constructed by the following procedure: Let $x$ be any defining function for $\partial M$, i.e. a function satisfying $x \in C^{\infty}(\bar{M}), x \geq 0, x(p)=0 \Leftrightarrow p \in \partial M$, with $d x$ non-vanishing at $\partial M$. Let $A^{i j} \in C^{\infty}(\bar{M})$ be symmetric traceless and let $X$ be any solution of the equation

$$
D_{i}\left[x^{-3}\left(D^{i} X^{j}+D^{j} X^{i}-\frac{2}{3} D_{k} X^{k} g^{i j}\right)\right]=-D_{i}\left(x^{-2} A^{i j}\right),
$$

define

$$
L^{i j} \equiv \frac{\omega^{2}}{x^{3}}\left(D^{i} X^{j}+D^{j} X^{i}-\frac{2}{3} D_{k} X^{k} g^{i j}\right)+\frac{\omega^{2}}{x^{2}} A^{i j}
$$

where $\omega$ is a solution of the equation

$$
\omega \Delta_{g} \omega-\frac{3}{2}|D \omega|_{g}^{2}+\frac{1}{4} \omega^{2}\left(R(g)-|L|_{g}^{2}\right)+\frac{3}{2}=0,
$$

satisfying $\omega \geq 0, \omega x^{-1}$ - uniformly bounded on $M$ from above and uniformly bounded away from zero, where $|L|_{g}^{2}=g_{i j} g_{k l} L^{i k} L^{j l}$. Setting

$$
\begin{aligned}
\tilde{g}_{i j} & =\omega^{-2} g_{i j}, \\
\tilde{K}^{i j} & =\omega^{3} L^{i j}+\varepsilon \tilde{g}^{i j},
\end{aligned}
$$

one obtains a solution of (2.2)-(2.1) satisfying (2.3). Moreover when $X$ is chosen appropriately (cf. point 1 below) the fields $\left(\tilde{g}_{i j}, \tilde{K}^{i j}\right)$ satisfy (in a rough way ${ }^{3}$ ) the asymptotic conditions appropriate to the "asymptotically hyperboloidal" setting (cf. $[9,1])$. In [1] the following has been shown:

1. For any $(g, A)$ as above one can find a unique solution $X \in x^{2} C^{\infty}(\bar{M})+$ $x^{4} \log x C^{\infty}(\bar{M})$ of (2.4) such that

$$
\left(\frac{x}{\omega}\right)^{2} L^{i j}=U^{i j}+x^{2} \log x U_{\log }^{i j}, \quad U^{i j}, U_{\log }^{i j} \in C^{\infty}(\bar{M}) .
$$

Given any $g$ there exists an open dense set (in the $C^{\infty}(\bar{M})$ topology) of $A$ 's for which $\left.U_{\log }^{i j}\right|_{\partial M} \not \equiv 0$ (however, there exists an infinite dimensional closed subspace of $A$ 's for which $\left.U_{\log }^{i j}\right|_{\partial M} \equiv 0$ ). If $\left.U_{\log }^{i j}\right|_{\partial M} \equiv 0$, then $U_{\log }^{i j} \equiv 0$ and

\footnotetext{
3 For a precise description of various conditions imposed, cf. [1]. In the smooth seed fields context discussed here, the boundary condition on $X$ is that $x^{-2} X$ be bounded; this ensures existence and uniqueness; cf. also footnote 2
} 
thus $x^{2} \omega^{-2} L^{i j} \in C^{\infty}(\bar{M})$. Let us note for further reference that in an orthonormal frame $e_{i}$, as described in Appendix A, if we write, in a neighbourhood of $\partial M$,

$$
L^{i j}=L_{0}^{i j}(v)+x L_{1}^{\imath \jmath}(v)+\ldots,
$$

where $\{v\}$ are coordinates on $\partial M$, then we have $L_{0}^{1 i} \equiv 0$, while both $L_{0}^{A B}(v)-$ $\frac{1}{2} L_{0}^{C D} h_{C D} h^{A B}$ and $L_{1}^{A B}(v)-\frac{1}{2} L_{1}^{C D} h_{C D} h^{A B}$ are freely specifiable tensor fields on $\partial M$.

2. For any $(g, A)$ as above one can find a solution $\omega \in \mathscr{A}_{\text {phg }}$, where $\mathscr{A}_{\text {phg }}$ denotes the space of polyhomogeneous functions on $\bar{M}$ (cf. Appendix E), of Eq. (2.6). More precisely there exists a sequence $\left\{N_{\jmath}\right\}_{j=0}^{\infty}$ with $N_{0}=N_{1}=N_{2}=N_{3}=0$, $N_{4}=1$ and functions $\omega_{i, j} \in C^{\infty}(\bar{M})$ such that

$$
\omega \sim \sum_{i \geq 0} \sum_{j=0}^{N_{\imath}} \omega_{\imath, j} x^{i} \log ^{j} x
$$

(cf. Appendix E for the meaning of “ "). For an open dense set of $(g, A)$ 's we have $\left.\omega_{4,1}\right|_{\partial M} \neq 0$. If $\left.\omega_{4,1}\right|_{\partial M} \equiv 0$, then $\omega \in C^{\infty}(\bar{M})$.

When $L^{i j} \equiv 0$, the obstruction to smoothness of $\omega$ has been analyzed in detail in [2]. In that reference it has been shown in particular that $\left.\omega_{4,1}\right|_{\partial M}$ vanishes if the Weyl tensor of the unphysical space-time metric is bounded near $\partial M$ (cf. [2] for details). It is the object of this paper to extend that analysis to the case $L^{i j} \not \equiv 0$.

\section{Weyl Tensor vs. Log Terms}

In this section we shall show that under some conditions the vanishing of the Weyl tensor at $\partial M$ is equivalent to the vanishing of some of the log terms in the solutions of the constraint equations. The methods of this section do not require any understanding of the origin of the log terms, which will be discussed in more detail in the next section. It should be stressed that we do not assume the condition $\operatorname{tr} \tilde{K}=$ const (which has been made in [1], as described in Sect. 2). To obtain sharp results we will consider functions which satisfy some detailed differentiability requirements near the conformal boundary, cf. Definition 3.1 below. The reader who is not interested in sharp results might assume that all the functions appearing in the formulas below are polyhomogeneous, thus all the coefficients of expansions in terms of powers of $x$ and $\log x$ are smooth functions of the boundary variables.

Let $(M, \tilde{g}, \tilde{K})$ be an initial data set, thus $M$ is an (open) manifold, $\tilde{g}$ is a Riemannian metric on $M$ and $\tilde{K}$ is a symmetric tensor field on $M$, with $(\tilde{g}, \tilde{M})$ satisfying the constraint equations (2.1)-(2.2). We shall also assume that $M=\operatorname{int} \bar{M}$, where $\bar{M}$ is a compact manifold with boundary, and that there exists a function $\omega \in C^{0}(\bar{M}) \cap C_{\mathrm{loc}}^{3}(M)$ such that

$$
\begin{gathered}
\tilde{g}=\omega^{-2} g \text { on } M, \\
\left.\omega\right|_{\partial M}=0,
\end{gathered}
$$

and $g$ is a Riemannian metric on $\bar{M}$ such that $g \in C^{0}(\bar{M}) \cap C_{\mathrm{loc}}^{3}(M)$. If we assume moreover that $\tilde{g}_{i j} \in H_{\mathrm{loc}}^{k+1}(M), \tilde{K}^{\imath \jmath} \in H_{\mathrm{loc}}^{k}(M)$, with some $k \geq 2$, then it follows from well known theorems $[14,13,4,5]$ that there exists a vacuum space-time $(V, \tilde{\gamma})$, 
with $\tilde{\gamma} \in C_{\text {loc }}^{k-1}(V)$, together with an embedding $i: M \rightarrow V$ such that $i^{*} \tilde{\gamma}=\tilde{g}$, and $\tilde{K}$ represents the extrinsic curvature tensor of $i(M)$ in $(V, \tilde{\gamma})$. Given an orthonormal frame $\tilde{e}_{i}, i=1,2,3$, for $\tilde{g}$ the four vectors $\left(\tilde{e}_{a}\right), a=0, \ldots, 3$, where $\tilde{e}_{0}=\tilde{\eta}$, the unit normal to $i(M)$ in $(V, \tilde{\gamma})$, form an orthonormal tetrad for $\tilde{\gamma}$. Let $\Omega$ be any function on $V$ such that

$$
\Omega \circ i=\omega
$$

define

$$
\gamma=\Omega^{2} \tilde{\gamma}
$$

In what follows we shall identify $M$ with $i(M)$ without explicitly mentioning this fact, and we shall always assume that (3.1) holds.

Following Geroch [12] we define

$$
\begin{gathered}
S_{a b}=R_{a b}(\gamma)-\frac{1}{6} R(\gamma) \gamma_{a b}, \\
\mathscr{G}^{a}{ }_{b}=R^{a}{ }_{b}(\tilde{\gamma})-\frac{1}{6} R(\tilde{\gamma}) \delta^{a}{ }_{b},
\end{gathered}
$$

where $R_{a b}(f), R(f)$ denote the Ricci tensor and the Ricci scalar of the metric $f$; we have [12]

$$
R_{a b c d}=C_{a b c d}+\gamma_{a[c} S_{d] b}-\gamma_{b[c} S_{d] a},
$$

where $R_{a b c d}$ and $C_{a b c d}$ are the Riemann tensor and the Weyl tensor of the metric $\gamma_{a b}$, and

$$
\begin{gathered}
S_{a b}=-\frac{2}{\Omega} \nabla_{a} \nabla_{b} \Omega+\frac{\nabla^{c} \Omega \nabla_{c} \Omega}{\Omega^{2}} \gamma_{a b}+\frac{1}{\Omega^{2}} \mathscr{G}_{a b}, \quad\left(\mathscr{G}_{a b} \equiv \gamma_{a c} \mathscr{G}_{b}^{c}\right), \\
C_{a b c d} \nabla^{d} \Omega+\Omega \nabla_{[a} S_{b] c}=\nabla_{[a}\left(\Omega^{-1} \mathscr{G}_{b] c}\right)-\Omega^{-2} \gamma_{c[a} \mathscr{G}_{b] d} \nabla^{d} \Omega \\
\nabla^{m}\left(\Omega^{-1} C_{a b c m}\right)=-\Omega^{-2} \nabla_{[a}\left(\Omega^{-1} \mathscr{G}_{b] c}\right)+\Omega^{-4} \gamma_{c[a} \mathscr{G}_{b] d} \nabla^{d} \Omega
\end{gathered}
$$

(in this section ${ }^{4}$ and in Appendices $\mathrm{A}$ and $\mathrm{D}$ all indices are tetrad indices, and are raised and lowered with $\gamma_{a b}=\operatorname{diag}(-1,1,1,1)$ ). If $\tilde{\gamma}$ is vacuum, then clearly $\mathscr{G}^{c}{ }_{b}=0$.

We wish to stress that above and throughout the remainder of this section we have not assumed that any kind of well behaved conformal completion of $(V, \gamma)$ exists, and that Eq. (3.5)-(3.7) hold whatever the extension $\Omega$ of $\omega$ to $V$.

The trace of Eq. (3.5) gives

$$
S \equiv \gamma^{a b} S_{a b}=\frac{4|\nabla \Omega|_{\gamma}^{2}}{\Omega^{2}}-\frac{2}{\Omega} \square \Omega+\frac{1}{\Omega^{2}} \gamma^{a b} \mathscr{G}_{a b} .
$$

Let us assume that near a connected component $\partial M_{i}$ of $\partial M$, we have

$$
\omega \in C^{1}(\bar{M}), \quad \gamma^{a b} \mathscr{G}_{a b}=o(1),
$$

and that we have chosen $\Omega$ so that along $M$ we have

$$
\square \Omega=o\left(\omega^{-1}\right) \text {. }
$$

The requirement that $S=o\left(\Omega^{-2}\right)$ gives

$$
\lim _{M \ni p \rightarrow \partial M_{i}}|\nabla \Omega|_{\gamma}^{2}=\left.0 \Rightarrow \omega_{0}\right|_{\partial M_{\imath}}= \pm\left.|D \omega|_{g}\right|_{\partial M_{i}}
$$

$\overline{4}$ On the other hand it should be noticed that in Sect. 4, 5, and in Appendix B coordinate indices are used 
where

$$
\left.\omega_{0} \equiv \nabla_{0} \Omega\right|_{M}
$$

It follows that if we wish $S_{a b}$ to be $o\left(\Omega^{-2}\right)$ we need to choose the boundary value of $\left.\nabla_{0} \Omega\right|_{\partial M_{i}}$ so that (3.9) holds. The trace free part of (3.5) reads

$$
S_{a b}-\frac{1}{4} S \gamma_{a b}=-\frac{2}{\Omega}\left[\nabla_{a} \nabla_{b} \Omega-\frac{1}{4} \square \Omega \gamma_{a b}\right]+\frac{1}{\Omega^{2}}\left(\mathscr{G}_{a b}-\frac{1}{4} \gamma^{c d} \mathscr{G}_{c d} \gamma_{a b}\right)
$$

Equation (3.10) shows that if $\mathscr{G}_{a b}=o(\Omega)$, then a necessary condition for $(V, \tilde{\gamma})$ to admit a Scri with a $C^{2}(\bar{V})$ metric is, that there exists on $M$ functions $\omega_{0}, \omega_{00}$ such that if we set

$$
\left.\nabla_{0} \Omega\right|_{M},\left.\quad \nabla_{0} \nabla_{0} \Omega\right|_{M}=\omega_{00}
$$

then we have

$$
\lim _{M \ni p \rightarrow \partial M_{\imath}}\left(\nabla_{a} \nabla_{b} \Omega-\frac{1}{4} \square \Omega \gamma_{a b}\right)=0
$$

By an abuse of terminology we shall refer to (3.11) as the statement, that a smooth Scri is shear free in the "gauge" $\square \Omega=0$.

Let us recall, that the couples $(\gamma, \Omega)$ and $\left(\phi^{2} \gamma, \phi \Omega\right)$ determine the same physical metric $\tilde{\gamma}=\Omega^{-2} \gamma$. If $\phi$ is a twice differentiable function uniformly bounded from above on $M$ and uniformly bounded away from zero on $M$, then a straightforward calculation shows that the condition (3.11) is invariant under such rescalings. As will be seen in Proposition 3.3, if the space-time under consideration admits a Scri which is a null hypersurface, then the geometric meaning of (3.11) is the vanishing of the conformal extrinsic curvature of Scri, as defined in Appendix C.

We wish to show that from the Cauchy problem point of view the requirement that Eq. (3.11) holds will lead to a constraint on the Cauchy data: a simple $3+1$ decomposition of (3.5) gives

$$
\begin{aligned}
S_{i j}-\frac{1}{3} g^{k l} S_{k l} g_{i j}= & -\frac{2}{\Omega}\left[\left(K_{i j}-\frac{1}{3} K g_{\imath j}\right) \nabla_{0} \Omega+\left(D_{i} D_{j} \Omega-\frac{1}{3} \Delta_{g} \Omega g_{i j}\right)\right] \\
& +\frac{1}{\Omega^{2}}\left(\mathscr{G}_{i j}-\frac{1}{3} g^{k l} \mathscr{G}_{k l} g_{i j}\right),
\end{aligned}
$$

where $K_{i j}$ is the extrinsic curvature of $M$ in the (unphysical) space-time $(V, \gamma)$, $K=g^{k l} K_{k l}$ and where $\Delta_{g}$ is the Laplacian of the metric $g_{i \jmath}$. Equation (3.12) implies:

Proposition 3.1. Suppose that $g \in C^{1}(\bar{M}), \omega \in C^{2}(\bar{M}), K \in C^{0}(\bar{M}), \mathscr{G}_{i j}=o(\Omega)$. A necessary condition for the existence of a conformal completion $\bar{V}$ of $(V, \gamma)$ such that $\gamma \in C^{2}(\bar{V})$ is that

$$
\begin{aligned}
\left.\left(K_{i \jmath}-\frac{1}{3} K g_{i j}\right)\right|_{\partial M} & =-\left.\varepsilon\left[\frac{1}{|D \omega|_{g}}\left(D_{i} D_{j} \omega-\frac{1}{3} \Delta_{g} \omega g_{\imath j}\right)\right]\right|_{\partial M}, \\
\varepsilon & \left.\equiv\left(\frac{\omega_{0}}{|D \omega|_{g}}\right)\right|_{\partial M}= \pm 1,
\end{aligned}
$$

where $D_{\imath}$ is the Levi-Civita connection of $g_{i j}$. 
It is worthwhile to analyze the covariance properties of Eq. (3.13): recall that the quadruplets $\left(\omega, g_{i j}, K^{i j},\left.\nabla_{0} \Omega\right|_{M}\right)$ and $\left(\bar{\omega}, \bar{g}_{i j}, \bar{K}^{i j}, \bar{\nabla} \bar{\Omega}_{M}\right)$, where

$$
\begin{gathered}
\bar{\omega}=\phi \omega \\
\bar{g}_{i j}=\phi^{2} g_{i \jmath} \\
\bar{K}^{i \jmath}=\phi^{-3}\left(K^{\imath j}-\frac{K}{3} g^{i \jmath}\right)+\left(\phi^{-1} \frac{K}{3}-\phi^{-2} \phi_{0}\right) g^{i j} \\
\left.\bar{\nabla}_{0} \bar{\Omega}\right|_{M}=\left.\nabla_{0} \Omega\right|_{M}+\phi^{-1} \phi_{0} \omega,
\end{gathered}
$$

with $\phi \in C_{\mathrm{loc}}^{2}(M), \phi_{0} \in C_{\mathrm{loc}}^{1}(M)$, determine the same physical Cauchy data set $(\tilde{g}, \tilde{K})$. If $\phi$ is assumed to be uniformly bounded from above on $M$ and uniformly bounded away from zero on $M$, then a straightforward calculation shows that the condition (3.13) is invariant under (3.14)-(3.17). Thus, (3.13) is indeed a condition on the physical Cauchy data.

Equation (3.13) is a non-trivial condition, because it is not satisfied by generic initial data as constructed in [1]:

Proposition 3.2. For generic $(g, A) \in C^{\infty}(\bar{M}) \oplus C^{\infty}(\bar{M})$, the solution of the constraint equations constructed by the conformal method will not satisfy (3.13).

Remarks. 1. Equation (3.12) shows that $S_{i j}$ will blow up at $\mathscr{T}=\bar{V} \backslash V$ as $\Omega^{-1}$ when (3.13) does not hold. This implies immediately that initial data for which (3.13) does not hold will lead to a space-time $(V, \tilde{\gamma})$ which does not admit compactifications $(V, \gamma)$ with a metric $\gamma \in C^{2}(\bar{V})$. It will be seen in Proposition 5.1 below that a stronger result holds: whenever (3.13) fails no conformal completion of the space-time with a polyhomogeneous Scri can exist, cf. Sect. 5 for details.

2. If $V$ admits a boundary such that $\mathscr{T}=\bar{V} \backslash V$ is a null hypersurface with $\partial M_{2} \subset \mathscr{T}$, then Eq. (3.18) below is equivalent to the vanishing of the conformal extrinsic curvature of $\mathscr{T}$ on $\partial M_{i}$ (cf. Appendix C). This together with Proposition 3.3 shows that in vacuum Eq. (3.11) is equivalent to the vanishing of the conformal extrinsic curvature of $\mathscr{T}$ on $\partial M_{i}$.

Proof. $2+1$ decomposing (3.13) one finds

$$
\left.\left(K_{A B}-\frac{1}{2} h^{C D} K_{C D} h_{A B}\right)\right|_{\partial M_{\imath}}=\varepsilon\left(\lambda_{A B}-\frac{1}{2} \lambda h_{A B}\right),
$$

where

$$
\lambda \equiv h^{A B} \lambda_{A B},
$$

the indices $A, B$, etc. run from 2 to $3, h_{A B}$ is the metric induced on $\partial M_{i}$ by $g$, and $\lambda_{A B}$ is the extrinsic curvature of $\partial M_{\imath}$ in $(M, g)$. As can be seen from what is said in Sect. 2 (and is analyzed in some more detail in [1]), both sides of (3.18) assume (independently) arbitrary values as $(g, A)$ run over $C^{\infty}(\bar{M}) \oplus C^{\infty}(\bar{M})$, and the result follows.

In the Appendix B we show that Cauchy data for which (3.18) holds are asymptotically flat in a quite satisfactory sense. The question whether or not and in which sense a space-time in which (3.13) fails to hold deserves the name of asymptotically flat and/or asymptotically Minkowskian remains to be investigated (cf. [7] for a discussion of this question in a Bondi-Sachs framework).

In the remainder of this paper we shall be concerned with the case in which (3.13) holds. In order to do this we need to impose on $g$ and $K$ differentiability conditions 
consistent with the occurrence of log terms in the solutions of the constraint equations. In order to cover sitatuions in which finite differentiability of space-time fields is appropriate we introduce the following:

Definition 3.1. For $0<k \in \mathbb{N}$ the function $f \in C_{\mathrm{loc}}^{k}(M) \cap C^{0}(\bar{M})$ is said to satisfy the $k$-condition at $\partial M_{i}$, where $\partial M_{i}$ is a connected component of the boundary $\partial M$ of $M$, if in local coordinates $y=\left(x, v^{A}\right)$ as described in Appendix $E$ we have

$$
\begin{gathered}
0 \leq|\alpha| \leq k-1 \lim _{x \rightarrow 0} \partial_{y}^{\alpha}\left(x^{k} \log x f\right)=0, \\
|\alpha|=k \lim _{x \rightarrow 0} \partial_{v}^{\alpha}\left(x^{k} \log x f\right)=0, \\
\lim _{x \rightarrow 0} \frac{\partial_{x}^{k}\left(x^{k} \log x f\right)}{\log x}=\left.k ! f\right|_{\partial M_{\imath}} .
\end{gathered}
$$

Remarks. 1. A function $f$ satisfying

$$
\forall \quad 0 \leq|\alpha| \leq k, \quad\left|\partial_{y}^{\alpha} f\right|=o\left(x^{-|\alpha|} \log ^{-1} x\right),
$$

will also satisfy the $k$-condition. In particular if $f \in C^{k}(\bar{M})$, then $f$ satisfies the $k$-condition.

2. Function displaying the above behaviour at $\partial M_{\imath}$ appear naturally when studying the regularity of solutions of constraint equations near $\partial M_{i}$, under finite differentiability hypotheses on the "seed fields" used in the conformal method (cf. [1]).

3. Note that if $f$ satisfies the $k$-condition, then $x^{k} \log x f \in C^{k-1}(\bar{M})$, and $x^{k} f \in$ $C^{k}(\bar{M})$; moreover the function $x^{k} \log x f$ behaves under differentiation and passing to the limits at $\partial M_{i}$ in a way somewhat similar as it would if $f \in C^{k} \bar{M}$. An example of a function which is not in $C^{1}(\bar{M})$ but which satisfies the $k$-condition for all $k \geq 1$ is given e.g. by the functions $\log (x)^{-\alpha}$, with any $\alpha>0$. Functions of the form $\log (x)^{-\alpha} \phi(v / x), \alpha>1$, where $\phi$ is any smooth function compactly supported in a coordinate patch on $\partial M$ (with $v$-coordinates on a coordinate patch of $\partial M$ extended to a neighbourhood of $\partial M$ in any smooth way) also satisfy the $k$-condition for any $k$.

4. This is a condition concerning space-derivatives of $f$ only, no time derivatives are involved.

Before stating the main result of this section, Theorem 3.1, we need to describe a property of the initial data sets considered:

Proposition 3.3. Consider a Cauchy data set $(M, \tilde{g}, \tilde{K})$ for a vacuum space-time $(V, \tilde{\gamma})$ such that

$$
\begin{gathered}
\tilde{g}_{\imath j}=\omega^{-2} g_{\imath \jmath}, \quad g_{i j}=\hat{g}_{i j}+x^{3} \log x \hat{g}_{i j}^{\text {loc }}, \\
\tilde{L}^{i \jmath}=\omega^{3} L^{\imath \jmath}, \quad L^{\imath j}=\hat{L}^{i j}+x^{2} \log x \hat{L}_{\operatorname{loc}}^{i j}, \\
\tilde{K}=\hat{K}+x^{2} \log x \hat{K}_{\log }, \\
\omega=\hat{\omega}+x^{4} \log x \hat{\omega}^{\log },
\end{gathered}
$$

where

$$
\tilde{K} \equiv \tilde{g}_{i j} \tilde{K}^{\imath \jmath}, \quad \tilde{L}^{\imath j} \equiv \tilde{K}^{\imath j}-\frac{\tilde{K}}{3} \tilde{g}^{i \jmath}
$$

with $\hat{g}_{i j} \in C^{3}(\bar{M}), \hat{\omega} \in C^{4}(\bar{M}), \hat{L}^{i j}, \hat{K} \in C^{2}(\bar{M})$, and $\hat{\omega}^{\log }$ satisfies the 4-condition, $\hat{g}_{i j}^{\log }$ satisfies the 3 -condition, and $\hat{L}_{\log }^{i j}, \hat{K}_{\log }$ satisfy the 2-condition at $\partial M_{\imath}$. Suppose 
moreover that there exists $\varepsilon= \pm 1$ such that

$$
\begin{gathered}
\left.\left(\lambda_{A B}-\frac{\lambda}{2} h_{A B}\right)\right|_{\partial M_{\imath}}=\left.\varepsilon\left(K_{A B}-\frac{\kappa}{2} h_{A B}\right)\right|_{\partial M_{\imath}}, \\
\lambda \equiv h^{A B} \lambda_{A B}, \quad \kappa \equiv h^{C D} K_{C D} .
\end{gathered}
$$

Then:

1. There exists a function $\Omega \in C_{\mathrm{loc}}^{4}(V)$ satisfying $\left.\Omega\right|_{M}=\omega$ such that

$$
\begin{gathered}
\lim _{M \ni p \rightarrow \partial M_{\imath}}|\nabla \Omega|_{\gamma}^{2}=0, \\
\lim _{M \ni p \rightarrow \partial M_{i}}\left(\nabla_{a} \nabla_{b} \Omega-\frac{1}{4} \square \Omega \gamma_{a b}\right)=0 ;
\end{gathered}
$$

2. On $M$ there exist functions $\hat{R}_{a b c d} \in C^{1}(\bar{M})$ and $R_{a b c d}^{\log }$ satisfying the 1-condition such that

$$
\left.R_{a b c d}\right|_{M}=\hat{R}_{a b c d}+\Omega \log \Omega R_{a b c d}^{\log }
$$

(in particular

$$
\begin{gathered}
\left.C_{a b c d}\right|_{M}=\hat{C}_{a b c d}+\Omega \log \Omega C_{a b c d}^{\log }, \\
\left.S_{a b}\right|_{M}=\hat{S}_{a b}+\Omega \log \Omega S_{a b}^{\log },
\end{gathered}
$$

with $\hat{C}_{a b c d}, \hat{S}_{a b} \in C^{1}(\bar{M})$, and $C_{a b c d}^{\log }, S_{a b}^{\log }$ satisfy the 1-condition). $\hat{R}_{a b c d}$ and $R_{a b c d}^{\log }$, respectively $C_{a b c d}$ and $C_{a b c d}^{\log }$, can be chosen to have all the symmetries of a Riemann tensor, respectively of a Weyl tensor.

Remarks. 1. It should be stressed that we do not claim that the decompositions (3.21)(3.23) are preserved under evolution (although we find it likely that this will be the case, at least under the conditions of Sect. 2). Thus, in all further calculations we assume that $(3,21)-(3.23)$ hold on $M$ only.

2. Since we are working in a frame formalism in this section, when $\hat{g}_{\imath j}^{\log }=0$ the positioning of indices on e.g. $\hat{L}_{\log }^{i j}$ upstairs or downstairs does not affect the decomposition of $L^{i j}$ into the log part and the regular part. If $\hat{g}_{\imath j}^{\log } \neq 0$ (or if one uses a coordinate formalism as in some other sections) one should use e.g. an orthonormal frame for $\hat{g}$ in all the calculations (or use $\hat{g}$ to raise and lower indices). This will not affect the equality of leading order part of the covariant and contravariant log part of a tensor, but will only lead to differences between some lower order terms in the covariant as compared to contravariant log part. Throughout this paper we shall freely move the index "log" in the subscript or superscript position, writing e.g. $\hat{L}_{i j}^{\log }$ for $\hat{g}_{i k} \hat{g}_{j l} \hat{L}_{\log }^{k l}$, etc.

In the remainder of this paper we shall assume that the choice of the extension $\Omega$ of $\omega$ from $M$ to $V$ has been made in a way consistent with Proposition 3.3.

The proof of Proposition 3.3 is a straightforward $2+2$ decomposition, and can be found in the Appendix D. Our main result is the following:

Theorem 3.1. Suppose that the hypotheses of Proposition 3.3 hold and let $(V, \tilde{\gamma})$ be a Cauchy development of $(M, \tilde{g}, \tilde{K})$ such that $\tilde{\gamma} \in C_{\mathrm{loc}}^{3}(V)$. Let $\partial M_{i}$ be a connected component of $\partial M$ which is diffeomorphic to $S^{2}$. Then in any orthonormal frame such that $e_{0} \perp M$ we have

$$
\lim _{M \ni p \rightarrow \partial M_{i}} C_{a b c d}(p)=0,
$$


if and only if

$$
\lim _{M \ni p \rightarrow \partial M_{i}} C_{\log }^{010 A}(p)=-\frac{1}{2} \lim _{M \ni p \rightarrow \partial M_{\imath}} S_{\log }^{1 A}(p)=0 .
$$

Remarks. 1. We do not assume $\tilde{K}=$ const, in particular there might be log terms in an asymptotic expansion of $\tilde{K}$ at $\partial M_{i}$.

2. Cauchy data $(\tilde{g}, \tilde{K})$ such that $(3.18)$ holds, constructed by the conformal method from generic smooth seed fields, as described in Sect. 2, will be have near $\partial M$ in a way consistent with the above assumptions. In such a case one moreover has

$$
\hat{L}_{\log }^{A B}=-\frac{\hat{L}_{\log }^{11}}{2} h^{A B},
$$

with $\hat{g}_{2 j}^{\log }=0, \hat{g}_{i j} \in C^{\infty}(\bar{M}), \hat{L}^{i j} \in C^{\infty}(\bar{M})$, while $\hat{\omega}^{\log }$ and $\hat{L}_{\text {log }}^{i j}$ are "polyhomogeneous" near $\partial M_{i}$.

3. For $(\tilde{g}, \tilde{K}) \in H_{5}^{\text {loc }}(M) \oplus H_{4}^{\text {loc }}(M)$ there exist vacuum developments satisfying $\gamma \in C_{\mathrm{loc}}^{3}(V)$.

4. The proof below is a careful reexamination of Geroch's version of the Weyl tensor vanishing theorem of Penrose [12].

5. The condition that $(V, \tilde{\gamma})$ is vacuum can be replaced by the conditions

$$
\begin{gathered}
\left.\mathscr{G}_{a b}\right|_{M}=o\left(x^{2}\right), \\
{\left[\left.\nabla_{[a}\left(\Omega^{-1} \mathscr{G}_{b] c}\right]\right|_{M}=o\left(x^{2}\right),\right.} \\
{\left.\left[\gamma_{c[a} \mathscr{G}_{b] d} \nabla^{d} \Omega\right]\right|_{M}=o\left(x^{4}\right) .}
\end{gathered}
$$

6. It should be stressed that in Theorem 3.1 we do not assume any uniform bounds on derivatives normal to $M$ of $R_{a b c d}, \hat{R}_{a b c d}$, and $R_{a b c d}^{\log }$.

7. Our arguments below also show that for other topologies (3.25) and (3.24) are not equivalent: from what is said here and from the results of [1] it follows that there exist solutions of constraint equations with $\partial M_{\imath} \neq S^{2}$ which satisfy the hypotheses of Proposition 3.3 (in fact $\left(g, K, \omega, \omega_{0}\right)$ can even be chosen to be smooth up to $\partial M_{i}$ ) and for which the space-time Weyl tensor does not vanish on $\partial M_{\imath}$.

Theorem 3.1 follows immediately from Proposition 3.3 and from the following:

Lemma 3.1. Under the hypotheses of Proposition 3.3, the only non-vanishing components of $\left.C_{a b c d}\right|_{\partial M_{\imath}}$ are (up to permutations) $\left.C_{0 A 0 B}\right|_{\partial M_{\imath}},\left.C_{0 A 1 B}\right|_{\partial M_{\imath}}$, and $\left.C_{1 A 1 B}\right|_{\partial M_{i}}$; with

$$
\begin{gathered}
\lim _{M \ni p \rightarrow \partial M_{i}} C_{0 A 0 B}=\varepsilon \lim _{M \ni p \rightarrow \partial M_{\imath}} C_{0 A 1 B}=\lim _{M \ni p \rightarrow \partial M_{i}} C_{1 A 1 B}, \\
\varepsilon \equiv \lim _{M \ni p \rightarrow \partial M_{\imath}}\left[|D \omega|_{g}^{-1} \nabla_{0} \Omega\right]= \pm 1
\end{gathered}
$$

$\left.C_{0 A 0 B}\right|_{\partial M_{\imath}}$ is a symmetric traceless tensor field on $\partial M_{i}$ and satisfies the equation

$$
\mathscr{D}_{A}\left(\left.C^{0 A 0 B}\right|_{\partial M_{\imath}}\right)=-\lim _{M \ni p \rightarrow \partial M_{i}}\left[|D \omega|_{g} C_{\log }^{10 B 0}\right]=\frac{1}{2} \lim _{M \ni p \rightarrow \partial M_{\imath}}\left[|D \omega|_{g} S_{\log }^{1 B}\right],
$$

where $\mathscr{D}_{A}$ is the Levi-Civita connexion of the metric $h$ induced by $g$ on $\partial M_{i}$.

Proof. Let $\Omega, \hat{R}_{a b c d}$, etc., be as given by Proposition 3.3. From Eq. (3.6) with $a, b=i, j$ and from (3.22)-(3.23), (3.28)-(3.29) one obtains

$$
\lim _{M \ni p \rightarrow \partial M_{i}} C_{i \jmath c d} \nabla^{d} \Omega=0 \text {. }
$$


Consider the Bianchi identity

$$
-\nabla_{0} R_{a 0}+\nabla_{i} R_{a i}=\frac{1}{2} \nabla_{a} R \Leftrightarrow-\nabla_{0} S_{a 0}+\nabla_{i} S_{a i}=\nabla_{a} S .
$$

Equation (3.32) with $a=j$ and (3.23) immediately give

$$
\left.\nabla_{0} S_{0 j}\right|_{M}=O(\log x) .
$$

Equation (3.32) inserted in (3.6) with $a=c=0$ yields

$$
\begin{aligned}
{\left.\left[C_{0 i 0 j} \nabla^{j} \Omega\right]\right|_{M} } & =-\left.\left[\frac{\Omega}{2}\left(\nabla_{0} S_{i 0}-\nabla_{i} S_{0 i}\right)\right]\right|_{M}+o(1) \\
& =o(1)
\end{aligned}
$$

From this we can conclude that

$$
\lim _{M \ni p \rightarrow \partial M_{\imath}} C_{0 i 0 j} \nabla^{j} \Omega=0
$$

which implies

$$
\lim _{M \ni p \rightarrow \partial M_{\imath}} C_{0 \imath 01}=0
$$

Define $\varepsilon= \pm 1$ by $\left.\left(\nabla^{a} \Omega e_{a}\right)\right|_{\partial M_{\imath}}=\left.\left[|D \omega|_{g}\left(\varepsilon e^{0}+e^{1}\right)\right]\right|_{\partial M_{\imath}}\left(\Rightarrow \lim _{M \ni p \rightarrow \partial M_{\imath}} \nabla_{0} \Omega=\right.$ $\left.\left.\varepsilon|D \omega|_{g}\right|_{\partial M_{i}}\right)$. From (3.31) we obtain

$$
\begin{gathered}
\lim _{M \ni p \rightarrow \partial M_{\imath}} C_{\imath \jmath 01}=0 \\
\lim _{M \ni p \rightarrow \partial M_{i}} C_{i j 0 A}=\varepsilon \lim _{M \ni p \rightarrow \partial M_{\imath}} C_{i \jmath 1 A} .
\end{gathered}
$$

Equations (3.34)-(3.35) imply

$$
\lim _{M \ni p \rightarrow \partial M_{\imath}} C_{a b 01}=0 .
$$

Consider e.g.

$$
\begin{aligned}
\lim _{M \ni p \rightarrow \partial M_{i}} C_{0323} & =\lim _{M \ni p \rightarrow \partial M_{i}}\left[-C_{0020}+C_{0121}+C_{0222}+C_{0323}\right] \\
& =\lim _{M \ni p \rightarrow \partial M_{i}} C_{0}^{a} 2 a=0
\end{aligned}
$$

which shows that

$$
\lim _{M \ni p \rightarrow \partial M_{\imath}} C_{0 A B C}=0
$$

Equation (3.36) also gives now

$$
\lim _{M \ni p \rightarrow \partial M_{\imath}} C_{1 A B C}=0 .
$$

The identity (A.13) of Appendix A implies

$$
\lim _{M \ni p \rightarrow \partial M_{\imath}} C_{1 A 1 B}=\lim _{M \ni p \rightarrow \partial M_{\imath}} C_{0 A 0 B},
$$


so that one also obtains

$$
\begin{aligned}
\lim _{M \ni p \rightarrow \partial M_{\imath}} C_{2323} & =\lim _{M \ni p \rightarrow \partial M_{i}}\left[-C_{0303}+C_{1313}+C_{2323}+C_{3333}\right] \\
& =\lim _{M \ni p \rightarrow \partial M_{\imath}}\left[C^{a}{ }_{3 a 3}\right]=0 \\
& \Rightarrow \lim _{M \ni p \rightarrow \partial M_{\imath}} C_{A B C D}=0
\end{aligned}
$$

Define

$$
\hat{C}_{A B} \equiv \lim _{M \ni p \rightarrow \partial M_{\imath}} C_{0 A 0 B}=\lim _{M \ni p \rightarrow \partial M_{\imath}} \hat{C}_{0 A 0 B}
$$

From (3.34)-(3.41) it follows that all nontrivial information about $\lim _{M \ni p \rightarrow \partial M_{\imath}} C_{a b c d}$ is contained in $\hat{C}_{A B}$, since the only non-vanishing components of $\lim _{M \ni p \rightarrow \partial M_{\imath}} C_{a b c d}$ are $C_{0 A 0 B}, C_{1 A 0 B}, C_{1 A 1 B}$ (and appropriate permutations of the indices thereof), with

$$
\lim _{M \ni p \rightarrow \partial M_{\imath}} C_{0 A 0 B}=\varepsilon \lim _{M \ni p \rightarrow \partial M_{\imath}} C_{0 A 1 B}=\lim _{M \ni p \rightarrow \partial M_{\imath}} C_{1 A 1 B}=\hat{C}_{A B} .
$$

Let us also note that we have

$$
\begin{aligned}
h^{A B} \hat{C}_{A B} & =\lim _{M \ni p \rightarrow \partial M_{\imath}}\left[-C_{0000}+C_{1010}+C_{2020}+C_{3030}\right] \\
& =\lim _{M \ni p \rightarrow \partial M_{\imath}} C^{a}{ }_{0 a 0}=0
\end{aligned}
$$

so that $\hat{C}_{A B}$ is a symmetric traceless tensor on $\partial M_{\imath}$. From (3.7) and (3.22) it follows

$$
\lim _{M \ni p \rightarrow \partial M_{\imath}}\left[\Omega \nabla_{a}\left(\Omega^{-1} \hat{C}^{a 0 B 0}\right)\right]=-\lim _{M \ni p \rightarrow \partial M_{\imath}}\left[C_{\log }^{a 0 B 0} \nabla_{a} \Omega\right]
$$

since this equation does not involve derivatives of $C^{a b c d}$ in directions normal to $M$. For $p \in \partial M_{\imath}$ let $\gamma_{p}=\gamma_{p}(s)$ be an integral curve of $e^{1}$ with $\gamma_{p}(0)=p$, thus for any function $f$ we have $\frac{d}{d s} f(\gamma(s))=e_{1}(f)(\gamma(s))$. From l'Hospital rule we have

$$
\begin{aligned}
\lim _{s \rightarrow 0}\left(\frac{\nabla_{a} \Omega \hat{C}^{a 0 B 0}}{\Omega}\right)(\gamma(s)) & =\lim _{s \rightarrow 0}\left[\frac{e_{1}\left(e_{a}(\Omega)\right) \hat{C}^{a 0 B 0}+\nabla_{a} \Omega e_{1}\left(C^{a 0 B 0}\right)}{e_{1}(\Omega)}\right](\gamma(s)) \\
& =\lim _{s \rightarrow 0}\left[e_{1}\left(\hat{C}^{10 B 0}\right)+\frac{e_{1}\left(e_{a}(\Omega)\right) \hat{C}^{a 0 B 0}}{e_{1}(\Omega)}\right](\gamma(s))
\end{aligned}
$$

Equation (3.43) shows that (3.42) does not involve derivatives of $\hat{C}^{a 0 B 0}$ in directions transverse to $\partial M_{i}$. A somewhat lengthy but otherwise straightforward $2+2$ decomposition of (3.43) gives

$$
\begin{aligned}
\lim _{s \rightarrow 0}\left[\Omega \nabla_{a}\left(\Omega^{-1} \hat{C}^{a 0 B 0}\right)\right]=\mathscr{D}_{A} \hat{C}^{A B} \\
\quad-\lim _{s \rightarrow 0}\left\{\frac{1}{e_{1}(\Omega)}\left[e_{1}\left(e_{A}(\Omega)\right)+\nabla_{0} \Omega \omega_{A}{ }^{0}{ }_{1}+\nabla_{1} \Omega \omega_{A}{ }^{1}{ }_{1}\right]\right\} \hat{C}^{A B},
\end{aligned}
$$

where $\mathscr{D}_{A}$ is the Levi-Civita connexion of the metric $h$ induced by $g$ on $\partial M_{\imath}$. Since $\lim _{M \ni p \rightarrow \partial M_{2}} \nabla_{1} \nabla_{A} \Omega=0,(3.42)$ gives

$$
\mathscr{D}_{A} \hat{C}^{A B}=-\lim _{M \ni p \rightarrow \partial M_{\imath}}\left[|D \omega|_{g} C_{\log }^{10 B 0}\right]
$$


To finish the proof, let us note that (3.22) inserted in (3.6) gives

$$
\left.\left(C_{0 A 0 d}^{\log } \nabla^{d} \Omega\right)\right|_{\partial M_{\imath}}=-\frac{1}{2} \lim _{M \ni p \rightarrow \partial M_{i}}\left(\frac{1}{\log \Omega} \nabla_{0} S_{A 0}\right) .
$$

From the Bianchi identity (3.32) we have

$$
\nabla_{0} S_{A 0}=\nabla_{\imath} S_{A i}-\nabla_{A} S
$$

so that from (3.23) one obtains

$$
\begin{aligned}
\lim _{M \ni p \rightarrow \partial M_{i}}\left(\frac{1}{\log \Omega} \nabla_{0} S_{A 0}\right) & =\lim _{M \ni p \rightarrow \partial M_{i}}\left(\frac{1}{\log \Omega} \nabla_{1} S_{A 1}\right) \\
& =\left.\left(|D \omega|_{g} S_{1 A}^{\log }\right)\right|_{\partial M_{\imath}} .
\end{aligned}
$$

Equations (3.45) and (3.46) give

$$
\left.C_{0 A 01}^{\log }\right|_{\partial M_{i}}=-\left.\frac{1}{2} S_{1 A}^{\log }\right|_{\partial M_{i}}
$$

which achieves the proof.

Proof of Theorem 3.1. Clearly, Eq. (3.44) shows that $\hat{C}^{A B}$ can vanish only if $C_{\log }^{10 B 0}$ does. If $\partial M_{i} \sim S^{2}$, the converse is also true because in the case the operator $L^{A B} \rightarrow \mathscr{D}_{A} L^{A B}$ which maps symmetric trace free tensors to vector fields is elliptic with trivial kernel.

Theorem 3.1 holds under more general conditions than those discussed in Sect. 2 , since it allows for non-constant $\tilde{K}$, and for $x^{3} \log x$ terms in $g_{\imath \jmath}$; moreover the structure of the log terms in $K_{i j}$ is much more general than that which is obtained in the setting of Sect. 2. When $g_{\imath \jmath} \in C^{3}(\bar{M})$, the $\left.C_{\log }^{01 A 0}\right|_{\partial M_{i}}$ terms responsible for the non-vanishing of the Weyl tensor at $\partial M_{i}$ can be related to the log terms in $K_{i j}$ as follows:

Corollary 3.1. Under the conditions of Theorem 3.1, suppose moreover that $\hat{g}_{i j}^{\log } \equiv 0$ (thus $g_{i j}=\hat{g}_{\imath j} \in C^{3}(M)$ ), and

$$
\omega_{0}=\hat{\omega}_{0}+\frac{1}{3 !} \omega_{0} \omega^{3} \log \omega \omega_{0}^{\log }
$$

$\hat{\omega}_{0} \in C^{3}(\bar{M}), \omega_{0}^{\log }$ satisfying the 3 -condition. If we write

$$
\begin{aligned}
\omega & =\hat{\omega}+\frac{1}{4 !} \omega^{4} \log \omega \omega^{\log }, \\
K_{i j} & =\hat{K}_{i j}+\frac{1}{2} \omega_{0} \omega^{2} \log \omega K_{i j}^{\log },
\end{aligned}
$$

$\left(\hat{\omega} \in C^{4}(\bar{M}), \hat{K}_{i j} \in C^{1}(\bar{M}), \omega_{\log }\right.$ satisfying the 4-condition, $K_{\imath j}^{\log }$ - satisfies the 1 condition), then

$$
\left.C_{\log }^{010 A}\right|_{\partial M_{i}}=\left.\frac{1}{2}\left(|D \omega|_{g} \omega_{0} K_{\log }^{1 A}\right)\right|_{\partial M_{\imath}}
$$

thus (3.24) holds iff $\left.K_{\log }^{1 A}\right|_{\partial M_{i}}=0$.

Proof. Inserting Eq. (3.49) into (3.5) one finds

$$
\left.S_{0 A}^{\log }\right|_{\partial M_{\imath}}=-\left.\left[|D \omega|_{g} \omega_{0} L_{1 A}^{\log }\right]\right|_{\partial M_{\imath}} .
$$


Theorem 3.1 and Corollary 3.1 show how the $K_{1 A}^{\log }$ terms affect the space-time geometry at $\partial M$. In the remainder of this section we shall consider the role of the other $\log$ terms. Let us start with an identity between the log terms in $\omega$ and $K_{i j}$ which holds under the conditions (3.27)-(3.29).

Lemma 3.2. Under the hypotheses of Corollary 3.1 we have the identity

$$
\left.\omega^{\log }\right|_{\partial M_{\imath}}=\left.\frac{1}{2}\left(K^{\log }-3 K_{11}^{\log }-\frac{4}{3} \tilde{K}^{\log }\right)\right|_{\partial M_{\imath}},
$$

with $\omega^{\log }$ as in (3.48), $K_{11}^{\log }$ as in (3.49), and

$$
\tilde{K}^{\log }=3\left(K^{\log }+\omega_{0}^{\log }\right) .
$$

Remark. Equation (3.53) is consistent with (D.11) which gives

$$
\tilde{K}=\hat{K}+\frac{1}{3 !} \omega_{0} \omega^{3} \log \omega \tilde{K}^{\log }, \quad \hat{K} \equiv 3 \hat{\omega}_{0}+\omega g^{i \jmath} \hat{K}_{i \jmath} .
$$

Thus $\tilde{K}^{\log }$ consists of the $\log$ terms in the trace of the extrinsic curvature of $M$ of the physical space-time metric. This implies in particular that $\tilde{K}^{\log }$ is invariant under the transformations (3.14)-(3.17) (this can also be checked by a direct calculation under appropriate hypotheses on $\phi$ and $\left.\phi_{0}\right)$.

Proof. Equation (3.7) gives

$$
\lim _{M \ni p \rightarrow \partial M_{\imath}}\left(\Omega \nabla_{a}\left(\Omega^{-1} \hat{C}^{a 0 c d}\right)\right)=-\lim _{M \ni p \rightarrow \partial M_{\imath}}\left(\nabla_{i} \omega C_{\log }^{20 c d}\right) .
$$

By Lemma 3.1 and a calculation similar to that of the proof of Lemma 3.1 one finds that the right-hand side of (3.54) with $c=0, d=1$ vanishes identically (this holds irrespectively of whether or not $\left.C_{\log }^{0 A B}\right|_{\partial M_{\imath}}=0$ ). From (3.54) one therefore obtains

$$
\left.C_{\log }^{0101}\right|_{\partial M_{\imath}}=0 \text {. }
$$

Equation (3.4) gives

$$
C_{0101}=R_{0101}+\frac{1}{2}\left(S_{11}-S_{00}\right)
$$

On the other hand we have

$$
\begin{aligned}
R_{0101} & =-S_{11}-\frac{1}{6} R+R_{\imath 1 \imath 1} \\
& \Rightarrow \\
C_{0101} & =-\frac{1}{2}\left(S_{00}+S_{11}+\frac{1}{3} R\right)+R_{\imath 1 \imath 1} .
\end{aligned}
$$

From (A.12) one obtains

$$
\left.C_{0101}^{\log }\right|_{\partial M_{\imath}}=-\left.\left(\frac{1}{2} h^{A B} S_{A B}^{\log }+S_{11}^{\log }\right)\right|_{\partial M_{\imath}},
$$

and (3.65)-(3.66) below give

$$
0=\left.C_{0101}^{\log }\right|_{\partial M_{\imath}}=\left.\left[\frac{|D \omega|_{g}^{2}}{2}\left(K_{11}^{\log }+K^{\log }+\frac{4}{3} \omega_{0}^{\log }+\frac{2}{3} \omega^{\log }\right)\right]\right|_{\partial M_{\imath}} .
$$

Equation (3.52) follows from (3.53) and (3.55).

Recall that the choice of $\omega_{0}^{\log }$ is arbitrary, so that for fixed $\tilde{K}^{\log }$ the function $K^{\log }$ is arbitrary. It follows that (3.52) imposes a relation between the functions $\omega^{\log }$ and 
$K_{11}^{\log }$ (determined by the seed fields if the conformal construction has been used) modulo choices of gauges. If, e.g., we have $\tilde{K}^{\log }=0$, we can choose $\omega_{0}^{\log }=0$ so that $K^{\log }=0$ and then Eq. (3.55) reads

$$
\left.\omega^{\log }\right|_{\partial M_{\imath}}=-\left.\frac{3}{2} K_{11}^{\log }\right|_{\partial M_{\imath}} .
$$

This last equation shows in particular, that under the conditions of Sect. 2 and with this choice of gauges the vanishing of all log terms in $K_{\imath j}$ implies no log terms in $\omega$ as well.

Theorem 3.2. Under the hypotheses of Proposition 3.3 let $(V, \tilde{\gamma})$ be a Cauchy development of $(M, \tilde{g}, \tilde{K})$ such that $\tilde{\gamma} \in C_{\log }^{3}(V)$; suppose moreover that $g_{i j} \in C^{3}(\bar{M})$ and

$$
\lim _{M \ni p \rightarrow \partial M_{\imath}} C_{a b c d}=0
$$

in an orthonormal frame such that $e_{0} \perp M$. Then the function $\omega$ can be extended from $M$ to a function $\Omega$ on $V$ (locally of the same differentiability class as $\omega$ ) such that

$$
\left.C_{a b c d}^{\log }\right|_{\partial M_{\imath}}=0
$$

if and only if

$$
\begin{gathered}
\left.\tilde{K}^{\log }\right|_{\partial M_{\imath}}=0, \\
\left.K_{1 A}^{\log }\right|_{\partial M_{i}}=0 \\
\left.\left(K_{A B}^{\log }-\frac{1}{2} h^{C D} K_{C D}^{\log } h_{A B}\right)\right|_{\partial M_{i}}=0 .
\end{gathered}
$$

Moreover if (3.58) holds then we also have

$$
\left.R_{a b c d}^{\log }\right|_{\partial M_{i}}=0 \text {. }
$$

Remark. When the topology of the boundary is spherical and $(\tilde{g}, \tilde{K})$ are constructed by the conformal method starting from smooth "seed fields," as described in Sect. 2, then the proof below shows that $\Omega^{-1} C_{a b c d}^{\log } \in C_{0}(\bar{M})$ and is polyhomogeneous if and only if (3.60)-(3.61) hold.

Proof. Regardless of the topology of $\partial M$ it follows from Eq. (3.30) that $\left.C_{01 A 0}^{\log }\right|_{\partial M_{i}}=$ $\left.S_{1 A}^{\log }\right|_{\partial M_{i}}=0$ whenever (3.57) holds, so that if we choose $\omega$ to be of the form (3.48) and $\omega_{0}$ to be of the form (3.47) then (3.60) will hold by (3.51). If we then choose $\omega_{00}$ so that

$$
\omega_{00}=\hat{\omega}_{00}+\frac{1}{2 !} \omega_{0}^{2} \omega^{2} \log \omega \omega_{00}^{\log }
$$

with $\hat{\omega}_{00} \in C^{2}(\bar{M}), \omega_{00}^{\log }$ satisfying the 2-condition, then the decomposition (3.49) inserted in (3.5) implies

$$
\begin{aligned}
& S_{0 i}^{\left.\log \right|_{\partial M_{i}}}=-\left.\left[|D \omega|_{g} \omega_{0}\left(K_{\imath 1}^{\log }+\omega_{0}^{\log } n_{i}\right)\right]\right|_{\partial M_{i}}, \\
& \left.S_{00}^{\log }\right|_{\partial M_{\imath}}=-\left.\left[|D \omega|_{g}^{2}\left\{\omega_{00}^{\log }+\frac{1}{3}\left(\omega^{\log }-\omega_{0}^{\log }\right)\right\}\right]\right|_{\partial M_{\imath}}, \\
& S_{\imath j}^{\left.\log \right|_{\partial M_{\imath}}}=\left.\left[|D \omega|_{g}^{2}\left\{-K_{i \jmath}^{\log }-\omega^{\log } n_{i} n_{j}+\frac{1}{3}\left(\omega^{\log }-\omega_{0}^{\log }\right) g_{i \jmath}\right\}\right]\right|_{\partial M_{\imath}} .
\end{aligned}
$$


Let us consider the $2+2$ decomposed form of (3.58). From (3.4) and (A.12) one obtains

$$
R_{1 A 1 B}=C_{1 A 1 B}+S_{A B}+\left.S_{11} h_{A B} \Rightarrow C_{1 A 1 B}^{\log }\right|_{\partial M_{\imath}}=-\left.\left(S_{A B}^{\log }+S_{11}^{\log } h_{A B}\right)\right|_{\partial M_{\imath}} .
$$

Equation (3.66) yields

$$
\left.C_{1 A 1 B}^{\log }\right|_{\partial M_{i}}=\left.\left[|D \omega|_{g}^{2}\left(K_{A B}^{\log }+\left(K_{11}^{\log }+\frac{1}{3} \omega^{\log }+\frac{2}{3} \omega_{0}^{\log }\right) h_{A B}\right)\right]\right|_{\partial M_{\imath}},
$$

and (3.55) shows that (3.58) with $a b c d=1 A 1 B$ is equivalent to (3.61). From (3.4), (A.11) and (3.66) with a little work one finds

$$
\left.C_{1 A 0 B}^{\log }\right|_{\partial M_{i}}=\left.\left[|D \omega|_{g} \omega_{0}\left(K_{A B}^{\log }+\frac{1}{2}\left(K_{11}^{\log }+\omega_{0}^{\log }\right) h_{A B}\right)\right]\right|_{\partial M_{\imath}},
$$

so that (3.61) and (3.53) imply that $\left.C_{1 A 0 B}^{\log }\right|_{\partial M_{i}}$ will vanish if and only if (3.59) holds. Manipulations with the Bianchi identities and Eq. (3.6) similar to those outlined at the end of the proof of Lemma 3.1 can be used to derive the following:

$$
\begin{gathered}
{\left.\left[|D \omega|_{g}\left(S_{A b}^{\log }+2 C_{1 A b 1}^{\log }\right)\right]\right|_{\partial M_{\imath}}=\left.\left.2\left[C_{1 A b 0}^{\log } \omega_{0}\right]\right|_{\partial M_{i}} \Rightarrow C_{1 A 01}^{\log }\right|_{\partial M_{i}}=-\left.\frac{1}{2} S_{A 0}^{\log }\right|_{\partial M_{\imath}} ;} \\
{\left.\left[C_{A B b d}^{\log } \nabla^{d} \Omega\right]\right|_{\partial M_{\imath}}=\left.0 \Rightarrow C_{A B C 0}^{\log }\right|_{\partial M_{i}}=\left.\varepsilon C_{A B C 1}^{\log }\right|_{\partial M_{\imath}},\left.\quad C_{A B 01}^{\log }\right|_{\partial M_{\imath}}=0 .}
\end{gathered}
$$

Calculations similar to the ones at the beginning of this proof show that all the remaining components of $\left.C_{a b c d}^{\log }\right|_{\partial M_{i}}$ vanish when (3.59)-(3.61) hold. Then Eq. (3.64) shows that $\left.S_{i 0}^{\log }\right|_{\partial M_{\imath}}=0$ provided we choose

$$
\left.\omega_{0}^{\log }\right|_{\partial M_{i}}=-\left.K_{11}^{\log }\right|_{\partial M_{\imath}}
$$

Similarly it follows from (3.64) that $\left.S_{00}^{\log }\right|_{\partial M_{i}}=0$ will hold if we set

$$
\left.\omega_{00}^{\log }\right|_{\partial M_{\imath}}=\left.\frac{1}{3}\left(\omega_{0}^{\log }-\omega^{\log }\right)\right|_{\partial M_{i}} .
$$

Equations (3.61) and (3.55) show now that the right-hand side of (3.66) vanishes identically, and the proof is completed.

\section{A Direct Approach}

Throughout this section, in contrast to Sect. 3, all indices are coordinate indices, and not tetrad indices. In this section we shall derive, by a direct analysis of the constraint equations, the obstructions to smoothness of the solutions of the constraint equations. The relationship between the "log terms" and the boundary values of the space-time Weyl tensor derived in the previous section will also be rederived here by a straightforward $2+2$ decomposition calculation. We will assume that the fields $g_{\imath \jmath}, L_{i \jmath}, \omega$ have the regularity described in Proposition 3.3 and that

$$
\tilde{K} \equiv 3 \varepsilon, \quad \varepsilon= \pm 1 .
$$

(If $\tilde{K} \equiv$ constant $\neq \pm 3$ the results described below go through with trivial modifications.) As in [2] (cf. Lemma 2.1 of [2]) it is possible to prove that the following gauge conditions can be imposed:

Proposition 4.1. Assume that $g \in C^{\infty}(\bar{M})$, that (4.1) holds, and that the hypotheses of Proposition 3.3 are satisfied. We may without loss of generality choose the coordinate system and the conformal factor so that 
1. the coordinates $\left(x, y^{2}, y^{3}\right)$ form a Gaussian coordinate system near $\partial M_{i}$, and

2. $\operatorname{tr} \lambda=0$ on $\partial M_{i}$.

The above and the scalar constraint equation imply then

$$
\omega=x+O\left(x^{3}\right) \text { near } \partial M_{i} .
$$

Remark. It is worthwhile pointing out that with the above conditions we have $|D x|_{g}=1$. Moreover we have the following useful identity on $\partial M_{\imath}$ :

$$
\lambda_{A B} \lambda_{C}^{B}=\frac{1}{2} \lambda: \lambda h_{A C}
$$

where we have used the notation

$$
\nu: \mu=\nu_{A B} \mu^{A B},
$$

where $\nu, \mu$ are covariant 2-tensors on $\partial M_{i}$. The identity (4.2) is easily proved using the fact that $\lambda_{A B}$ is symmetric and therefore may be diagonalized. Equation (4.2) implies in particular

$$
\mu^{A B} \mu_{B C} \nu_{A}^{C}=0
$$

for any symmetric trace free 2-tensors $\mu, \nu$ on $\partial M_{i}$. Similarly we will use the notation $\lambda: \mu: \nu=\lambda_{A}{ }^{B} \mu_{B}{ }^{C} \nu_{C}{ }^{A}$, etc.

We shall start our analysis with the vector constraint equation.

\subsection{The Vector Constraint Equation}

Consider the momentum constraint equation for the physical data,

$$
\tilde{D}^{i}\left(\tilde{K}_{\imath j}-\tilde{K} \tilde{g}_{i \jmath}\right)=0 \text {. }
$$

Setting

$$
\tilde{K}_{\imath \jmath}=\omega^{-1} L_{i j}+\frac{1}{3} \tilde{K} \tilde{g}_{i j}
$$

with $\tilde{K} \equiv$ constant, the momentum constraint equation takes the form

$$
\omega D^{i} L_{i \jmath}-2 \omega^{, i} L_{\imath j}=0 .
$$

A $2+1$ decomposition calculation yields

$$
\begin{aligned}
\left(x \partial_{x}-2-x \lambda_{F}{ }^{F}\right) L_{11}+x \mathscr{D}^{A} L_{1 A}+x \lambda^{A B} L_{A B} & =o\left(x^{2}\right) \\
\left(x \partial_{x}-2-x \lambda_{F}{ }^{F}\right) L_{1 B}+x \mathscr{D}^{A} L_{A B} & =o\left(x^{2}\right) .
\end{aligned}
$$

We have:

Proposition 4.2. Let the hypotheses and the gauge conditions of Proposition 4.1 hold. According to Proposition 3.3 we can write

$$
L_{i j}=\hat{L}_{i j}+x^{2} \log x \hat{L}_{i j}^{\log }
$$

with $\hat{L}_{\imath j} \in C^{2}(\bar{M})$ and $\hat{L}_{i j}^{\log }$ satisfying the 2-condition at $\partial M_{\imath}$. Suppose moreover that

$$
\left.\hat{L}_{A B}^{\log }\right|_{\partial M_{i}}=-\frac{1}{2} \hat{L}_{11}^{\log } h_{A B} .
$$


Then

$$
\begin{aligned}
\left.L_{1 \jmath}\right|_{\partial M_{\imath}} & =0 \\
\left.\partial_{x} L_{11}\right|_{\partial M_{\imath}} & =\lambda^{A C} L_{A C}, \\
\left.\partial_{x} L_{1 B}\right|_{\partial M_{i}} & =\mathscr{D}^{C} L_{C B},
\end{aligned}
$$

and the terms $\hat{L}_{1 j}^{\log }$ in the decomposition of $L_{i j}$ take the following values on $\partial M_{\imath}$ :

$$
\begin{aligned}
\left.\hat{L}_{11}^{\log }\right|_{\partial M_{\imath}}= & -\left(\mathscr{D}^{A} \mathscr{D}^{B} L_{A B}+\partial_{x} \lambda_{A B} L^{A B}+\lambda^{A B} \partial_{x} L_{A B}\right) \\
= & -\left(\mathscr{D}^{A} \mathscr{D}^{B} L_{A B}+R_{A B} L^{A B}+\lambda^{A B} \partial_{x} L_{A B}\right) \\
\left.\hat{L}_{1 A}^{\log }\right|_{\partial M_{\imath}}= & -\partial_{x}\left(\mathscr{D}^{B} L_{A B}\right) \\
= & -\left[\mathscr{D}^{B}\left(\partial_{x} L_{A B}\right)+2 \lambda^{B C} \mathscr{D}_{B} L_{A C}\right. \\
& \left.+2 L_{A B} \mathscr{D}^{C} \lambda_{C B}+L^{B C} \mathscr{D}_{A} \lambda_{B C}\right]
\end{aligned}
$$

where $R_{A B}$ denotes the pull-back of the Ricci tensor of $g_{i j}$ to $\partial M_{i}$.

Remarks. 1. The tensor $\left.\hat{L}_{i j}^{\log }\right|_{\partial M_{\imath}}$ defined above in Eq. (4.8) differs from the trace-free part of $\left.K_{\imath j}^{\log }\right|_{\partial M_{i}}$ defined in Eq. (3.49) by a normalization factor of $\varepsilon / 2$.

2. The hypothesis (4.9) holds for solutions of the constraint equations constructed by the conformal method starting from seed fields which are smooth up to the boundary.

Proof. The result is obtained by inserting the decomposition (4.8) in (4.6)-(4.7) and comparing terms with appropriate powers of $x$. To get (4.13) one uses the identity

$$
R_{A B}=\partial_{x} \lambda_{A B}+r_{A B}+2 \lambda_{A C} \lambda^{C}{ }_{B}-\lambda_{A B} \lambda_{C}^{C} \text {. }
$$

Note that $\hat{L}_{A B}^{\log }$ is expressed in terms of $\hat{L}_{11}^{\log }$. This means that the vanishing of $\hat{L}_{i j}^{\log }$ is equivalent to the vanishing of the three functions $\hat{L}_{11}^{\log }, \hat{L}_{1 A}^{\log }$ on $\partial M_{i}$.

In the remainder of this subsection we shall consider those solutions of the constraint equations which have been constructed by the conformal method starting from seed fields $g, A, x \in C^{\infty}(\bar{M})$, as described in Sect. 2. For simplicity we shall only consider those $A_{i j}$ 's for which we have

$$
\left.A_{i j} D^{i} x\right|_{\partial M_{i}}=0
$$

Equation (4.10) shows that any tensor $L_{i j}$ satisfying (4.5) necessarily satisfies (4.16). This observation and a simple argument using uniqueness of solutions of (2.4) shows that the space of transverse traceless tensors constructed as described in Sect. 2 is exhausted by those tensors which are obtained from tensors satisfying (4.16).) Under (4.16) it follows by comparing terms with appropriate powers of $x$ in (2.4) that the desired solution $X$ of (2.4) satisfies

$$
X=O\left(x^{3}\right) .
$$

Equations (2.5) and (4.17) show that we have

$$
\begin{aligned}
\left.\left(L_{A B}-\frac{1}{2} h^{C D} L_{C D} h_{A B}\right)\right|_{\partial M_{\imath}} & =\left.\left(A_{A B}-\frac{1}{2} h^{C D} A_{C D} h_{A B}\right)\right|_{\partial M_{\imath}}, \\
\left.\left(\partial_{x} L_{A B}-\frac{1}{2} h^{C D} \partial_{x} L_{C D} h_{A B}\right)\right|_{\partial M_{\imath}} & =\left.\left(\partial_{x} A_{A B}-\frac{1}{2} h^{C D} \partial_{x} A_{C D} h_{A B}\right)\right|_{\partial M_{\imath}} .
\end{aligned}
$$

It is natural to ask the question, for which seed fields will one obtain solutions of the constraint equations such that $L_{i j} \in C^{\infty}(\bar{M})$ - as discussed in Sect. 2, this will 
occur if $\left.\hat{L}_{i j}^{\log }\right|_{\partial M_{i}}=0$. The appropriate conditions on $A_{i j}$ can be derived by inserting (4.18)-(4.19) into (4.13)-(4.14), they do not, however, seem to be very enlightening in general. Some simplifications occur if in addition to (4.16) we assume that "Scri is shear free," in the sense that

$$
\left.L_{A B}\right|_{\partial M_{i}}=\varepsilon \lambda_{A B}
$$

with $\varepsilon= \pm 1$. Under these conditions the equation $\left.\hat{L}_{11}^{\log }\right|_{\partial M_{\imath}}=0$ can be rewritten as

$$
\left.\hat{L}_{11}^{\log }\right|_{\partial M_{\imath}}=-\left.\left[\lambda^{A B} \partial_{x} A_{A B}+\varepsilon\left(\mathscr{D}^{A} \mathscr{D}^{B} \lambda_{A B}+R_{A B} \lambda^{A B}\right)\right]\right|_{\partial M_{\imath}}=0 .
$$

With some work from (4.14) one finds that

$$
\left.\hat{L}_{1 A}^{\log }\right|_{\partial M_{i}}=-\mathscr{D}^{B} p_{A B}
$$

where

$$
p_{A B} \equiv \partial_{x} A_{A B}+\frac{3}{2} \varepsilon \lambda_{C D} \lambda^{C D} h_{A B} .
$$

From $L^{i}{ }_{i}=0$ and from (4.20) it is simple to show that $p_{A B}$ is trace-free, so that $p_{A B}$ is a symmetric traceless tensor on the two dimensional manifold $\partial M_{i}$. If $\partial M_{i}$ is a sphere, $\left.\hat{L}_{1 A}^{\log }\right|_{\partial M_{i}}=0$ implies $p_{A B}=0$; for other topologies this will, however, not be true in general.

\subsection{The Scalar Constraint Equation}

Under the conditions of Proposition 4.1 we can write

$$
\omega=x+x^{3} \omega_{3}+x^{4} \log (x) \omega_{4,1}+\text { l.o. },
$$

where l.o. denotes lower order terms. Inserting the expansion (4.24) in Eq. (2.6) one obtains

$$
\begin{aligned}
\left.\omega_{3}\right|_{\partial M_{i}} & =\frac{1}{12}\left[-4 \partial_{x}\left(\lambda_{F}{ }^{F}\right)-L_{A B} L^{A B}+R(g)\right], \\
\left.\omega_{4,1}\right|_{\partial M_{\imath}} & =\frac{1}{16}\left[2 \partial_{x}^{2}\left(\lambda_{F}{ }^{F}\right)+2 L^{A B} \partial_{x} L_{A B}+4 L_{A}^{C} L_{C}{ }^{B} \lambda^{A}{ }_{B}-\partial_{x} R\right] \\
& =\frac{1}{16}\left[2 \partial_{x}^{2}\left(\lambda_{F}{ }^{F}\right)+2 L^{A B} \partial_{x} L_{A B}-\partial_{x} R\right],
\end{aligned}
$$

where we have used (4.3) and the fact that $\left.L_{1 j}\right|_{\partial M_{i}}=0$ which implies that $\left.h^{A B} L_{A B}\right|_{\partial M_{i}}=0$. A $2+1$ decomposition of the second Bianchi identity $D_{i} R=$ $2 D^{j} R_{i \jmath}$ using $\lambda_{F}{ }^{F}=0$ on $\partial M_{i}$ gives the relation

$$
\left.\partial_{x} R\right|_{\partial M_{\imath}}=2\left(\partial_{x} R_{11}-\mathscr{D}^{A} \mathscr{D}^{B} \lambda_{A B}+\lambda^{A B} R_{A B}\right) .
$$

Note that since $r_{A B}=\frac{1}{2} r h_{A B},\left.\lambda_{F}{ }^{F}\right|_{\partial M_{i}}=0$ and $\left.\lambda_{A}{ }^{B} \lambda_{B}{ }^{C} \lambda_{C}{ }^{A}\right|_{\partial M_{\imath}}=0$, ' one finds from the identity (4.15) that $\left.\lambda^{A B} R_{A B}\right|_{\partial M_{\imath}}=\lambda: \partial_{x} \lambda$. Further, using $R_{11}=\partial_{x}\left(\lambda_{F}^{F}\right)-\lambda: \lambda$ we find

$$
\left.\partial_{x} R_{11}\right|_{\partial M_{\imath}}=\partial_{x}^{2}\left(\lambda_{F}^{F}\right)-2 \lambda^{A B} \partial_{x} \lambda_{A B},
$$

where we have used (4.3). Thus we get

$$
\omega_{4,1}=\left.\frac{1}{8}\left[R_{A B} \lambda^{A B}+\mathscr{D}^{A} \mathscr{D}^{B} \lambda_{A B}+\partial_{x} L: L\right]\right|_{\partial M_{\imath}},
$$


where in the last step we have used the fact that $g^{i j} L_{i j} \equiv 0$ and $\left.L_{1 j}\right|_{\partial M_{i}}=0$, which implies $\left.h^{A B} L_{A B}\right|_{\partial M_{\imath}}=0$ and therefore $\left.L_{A C} L^{C}{ }_{B}\right|_{\partial M_{i}}=\frac{1}{2} L_{C D} L^{C D} h_{A B}$. In particular, if "Scri is shear free" in the sense that (4.20) holds, then

$$
\omega_{4,1}=-\left.\frac{\varepsilon}{8} L_{11}^{\log }\right|_{\partial M_{i}} .
$$

This gives an alternative proof of Lemma 3.2, namely that under the present conditions the vanishing of $\left.L_{11}^{\log }\right|_{\partial M_{i}}$ implies that the boundary value of the log coefficient in $\omega$ vanishes as well.

\subsection{The Weyl Tensor}

Throughout this section we shall assume that the hypotheses and the gauge conditions of Proposition 4.1 are satisfied. By a transformation of the form (3.16) with an appropriate $\phi_{0}$ we can also achieve

$$
K=0 \text {, }
$$

and we shall also assume in this section that (4.30) holds. Due to $\operatorname{Ein}(\tilde{\gamma})=0, C_{20 k}{ }^{0}$ is the negative of the Ricci trace of the spatial part of $\operatorname{Riem}(\tilde{\gamma})$ :

$$
\begin{aligned}
-C_{i 0 k}{ }^{0}=-\tilde{C}_{i 0 k}{ }^{0} & =-\tilde{R}_{i 0 k}{ }^{0} \\
& =-\tilde{\gamma}_{\beta}^{\alpha} \tilde{R}_{i \alpha k}^{\beta}+\tilde{g}_{l}^{j} \tilde{R}_{i j k}{ }^{l} \\
& =\tilde{g}_{l}^{\jmath} \tilde{R}_{i j k}{ }^{l},
\end{aligned}
$$

where $C_{a b c}{ }^{d}$, respectively $\tilde{C}_{a b c}{ }^{d}$, denotes the Weyl tensor of the metric $\gamma$, respectively $\tilde{\gamma}$. Using the conformal transformation rules and the Gauss equation (A.12) we get the formula

$$
\begin{aligned}
C_{i j} \equiv & C_{0 i 0 j}=R_{\imath \jmath}(g)-\frac{1}{3} R(g) g_{i j} \\
& +\frac{1}{\omega}\left(D_{\imath} D_{\jmath} \omega-\frac{1}{3} D_{k} D^{k} \omega g_{\imath \jmath}+\varepsilon L_{i j}\right)-L_{\imath}{ }^{k} L_{k \jmath}+\frac{1}{3} L^{k l} L_{k l} g_{\imath \jmath}
\end{aligned}
$$

By l'Hospital's rule we have

$$
\left.\left[\frac{1}{\omega}\left(D_{A} D_{B} \omega+\varepsilon L_{A B}\right)\right]\right|_{\partial M_{i}}=-\partial_{x} \lambda_{A B}+\varepsilon \partial_{x} L_{A B}
$$

so that, using $\left.\lambda_{A}{ }^{A}\right|_{\partial M_{2}}=0,\left.L_{A}{ }^{A}\right|_{\partial M_{i}}=0$ and the fact that the square of a symmetric traceless two dimensional tensor is proportional to the metric one is led to

$$
\left.C_{A B}\right|_{\partial M_{\imath}}=R_{A B}-\partial_{x} \lambda_{A B}+\varepsilon \partial_{x} L_{A B}+\kappa h_{A B}
$$

for some function $\kappa$. Now we have $C_{0}{ }^{i}{ }_{0 i}=C_{0}{ }^{\alpha}{ }_{0 \alpha}=0$, and since $\left.C_{0101}\right|_{\partial M_{i}} \equiv 0$ by Sect. 3 it follows ${ }^{5}$ that $\kappa$ can be determined by the requirement that the right-hand

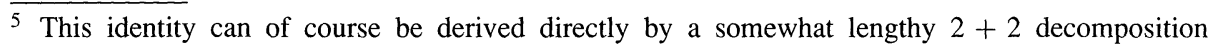
calculation 
side of (4.32) is trace-free. The embedding equation (4.15) allows us to rewrite Eq. (4.32) as

$$
\left.C_{A B}\right|_{\partial M_{\imath}}=\varepsilon\left(\partial_{x} L_{A B}-\frac{1}{2} h^{C D} \partial_{x} L_{C D} h_{A B}\right) .
$$

Thus, the value of $C_{A B}$ on $\partial M_{\imath}$ is just the trace free part of $\partial_{x} L_{A B}$. Equations (4.33) and (4.22)-(4.23) give an alternative proof of the assertion, that under the shear-free condition (4.20) the boundary values of the $\log$ terms in $L_{1 A}$ are determined by $\left.C_{a b c d}\right|_{\partial M_{i}}$.

Let us finally mention that with some work it follows from (4.31) that $\hat{C}_{i j}^{\log }$ takes the form

$$
\begin{aligned}
\left.\hat{C}_{11}^{\log }\right|_{\partial M_{\imath}} & =8 \omega_{4,1}+\varepsilon \hat{L}_{11}^{\log } \\
\left.\hat{C}_{1 A}^{\log }\right|_{\partial M_{i}} & =\varepsilon \hat{L}_{1 A}^{\log }, \\
\left.\hat{C}_{A B}^{\log }\right|_{\partial M_{i}} & =4 \omega_{4,1} h_{A B}-\varepsilon \hat{L}_{A B}^{\log }
\end{aligned}
$$

(cf. also (3.50)). We see from (4.29) and from Proposition 4.2 that under the hypotheses of this section the fields $\hat{C}_{11}^{\mathrm{log}}$ and $\hat{C}_{A B}^{\mathrm{log}}$ vanish identically on $\partial M_{\imath}$ (cf. also the proof of Lemma 3.2).

\section{The Evolution Problem}

The aim of this section is to discuss the question of existence of developments $(V, \tilde{\gamma})$ of the "hyperboloidal" Cauchy data $(\tilde{g}, \tilde{K})$ which admit some kind of conformal completion $(\bar{V}, \gamma)$. Let us start with smooth up to boundary initial data:

Theorem 5.1. Let $\partial M_{i} \approx S^{2}$, the two dimensional sphere, and let $(\tilde{g}, \tilde{K})$ be a solution of the vacuum constraint equations for which there exist smooth functions $\omega$ and $\omega_{0}$, with $\omega / x \in C^{\infty}(\bar{M})$, such that the fields

$$
\begin{gathered}
g_{\imath \jmath}=\omega^{2} \tilde{g}_{\imath \jmath} \\
K^{i \jmath}=\omega^{-3}\left(\tilde{K}^{\imath \jmath}-\frac{1}{3} \tilde{g}_{k l} \tilde{K}^{k l} \tilde{g}^{\imath \jmath}\right)+\frac{\tilde{g}_{k l} \tilde{K}^{k l}-3 \omega_{0}}{3 \omega} g^{i j}
\end{gathered}
$$

are in $C^{\infty}(\bar{M})$. Suppose moreover that there exists $\varepsilon= \pm 1$ such that

$$
\left.\left(\lambda_{A B}-\frac{h^{C D} \lambda_{C D}}{2} h_{A B}\right)\right|_{\partial M_{i}}=\left.\varepsilon\left(K_{A B}-\frac{h^{C D} K_{C D}}{2} h_{A B}\right)\right|_{\partial M_{\imath}} .
$$

Then

1. There exists a Lorentzian manifold with boundary $(\bar{V}, \gamma)$ and a function $\Omega \in$ $C^{\infty}(\bar{V})$ (defining function for $\partial V$ ) such that (int $\bar{V}, \Omega^{-2} \gamma$ ) is a development of $(M, \tilde{g}, \tilde{K})$, with $\partial M_{\imath}$ being a smooth submanifold of $\bar{V} \backslash V$.

2. If moreover $M=B(1)$ (the unit ball $\mathbb{R}^{3}$ ), then there exists $\varepsilon_{0}>0$ such that if

$$
\|\omega-\check{\omega}\|_{C^{8}(\bar{M})}+\left\|g_{i j}-\check{g}_{i j}\right\|_{C^{7}(\bar{M})}+\left\|\omega_{0}\right\|_{C^{7}(\bar{M})}+\left\|K^{i j}\right\|_{C^{6}(\bar{M})}<\varepsilon_{0},
$$

where $\breve{g}_{i j}$ is the standard flat metric on $B(1)$ and $\breve{\omega}=\left(1-r^{2}\right) / 2$, then (int $\bar{V}, \Omega^{-2} \gamma$ ) is timelike and null future geodesically complete.

Remarks. 1. The norm in (5.4) can be considerably weakened at the price, however, of a rather more complicated statement of the corresponding result, cf. [9] for details. 
2. Part 1 above still holds when $\partial M_{i} \not \approx S^{2}$ provided one adds the assumption that $\left.C_{0 A 0 B}\right|_{\partial M_{i}}=0$.

Proof. The calculation of Sect. 3 shows that $\left.\nabla_{0} \Omega\right|_{M} \in C^{\infty}(\bar{M})$ and $\left.\nabla_{0} \nabla_{0} \Omega\right|_{M} \in$ $C^{\infty}(\bar{M})$ can be chosen so that $\left.S_{a b}\right|_{M} \in C^{\infty}(\tilde{M}),\left.\Omega^{-1} C^{a}{ }_{b c d}\right|_{M} \in C^{\infty}(\bar{M})$. Point 1 follows then from Theorem 6.5 of [8], point 2 follows from Theorem 3.5 of [9] and from the results of [11].

The condition (5.3) can be rewritten in terms of the seed fields $(g, A)$, so that from (4.21)-(4.23), (3.56) and Theorem 5.1 one obtains:

Corollary 5.1. Let $\partial M \approx S^{2}$, the two dimensional sphere, and let $(\tilde{g}, \tilde{K})$ be constructed as described in Sect. 2 starting with smooth seed fields $(g, A)$. Suppose moreover that there exists $\varepsilon= \pm 1$ such that

$$
\left.\left(\lambda_{A B}-\frac{\lambda}{2} h_{A B}\right)\right|_{\partial M}=\left.\varepsilon\left[A_{A B}-\frac{1}{2} h^{C D} A_{C D} h_{A B}\right]\right|_{\partial M} .
$$

Without loss of generality we may choose the "gauge"

$$
\left.\lambda\right|_{\partial M_{\imath}}=0
$$

with $x, v^{A}$ - Gauss coordinates near the boundary. Suppose that moreover in this gauge we have

$$
\begin{gathered}
\left.A_{1 j}\right|_{\partial M}=0 \\
{\left.\left[\mathscr{D}^{A} \mathscr{D}^{B} \lambda_{A B}+R_{A B} \lambda^{A B}\right]\right|_{\partial M}=0} \\
\left.\left(\partial_{x} A_{A B}-\frac{1}{2} h^{C D} \partial_{x} A_{C D} h_{A B}\right)\right|_{\partial M}=0 .
\end{gathered}
$$

Then the fields $\omega$, and $K$ constructed by the conformal method are in $C^{\infty}(\bar{M})$. Further, $\omega^{-1} C^{a}{ }_{b c d}$ is in $C^{\infty}(\bar{M})$ and the conclusions of Theorem 5.1 hold.

Remark. It is clear from the methods of [1] that under the hypotheses of Corollary 5.1 the condition (5.4) can be replaced by the condition that $\left(g_{\imath j}, A^{i j}\right)$ are sufficiently close to $\left(\check{g}_{i j}, 0\right)$ in some possibly higher norm.

We shall now consider the case of initial data sets for which the log terms occur. Note first, that if $K^{i j}$ has $\log$ terms in its expansion at $\partial M$, then even if we start with $g_{i j} \in C^{\infty}(\bar{M})$ then by evolution $g_{i j}$ will pick up log terms at the next moment of time. It follows that the appropriate setup to discuss the evolution problem is that of polyhomogeneous space-time metrics $\gamma$. Before proceeding any further, let us intrdouce some terminology. Let $\overline{\mathscr{C}}$ be an $n$-dimensional connected, paracompact, Hausdorff manifold with boundary, thus $\mathscr{C} \equiv$ int $\overline{\mathscr{C}}$ and $\partial \mathscr{\mathscr { C }} \equiv$ $\partial \overline{\mathscr{C}} \equiv \overline{\mathscr{C}} \backslash \mathscr{M} b$ are smooth manifolds. A semi-Riemannian metric $f$ on $\overline{\mathscr{C}}$ will be called polyhomogeneous if $f \in C_{\mathrm{loc}}^{\infty}(\mathscr{\mathscr { C }}) \cap C^{0}(\overline{\mathscr{C}})$ and if in local coordinates $y=(x, v)$ near $\partial \mathscr{l}$, where $x$ is a defining function for $\partial \mathscr{l} b$ and $v$ are coordinates on $\partial \mathscr{C l}$, the functions $f_{\mu \nu} \equiv f\left(\partial / \partial y^{\mu}, \partial / \partial y^{\nu}\right)$ are polyhomogeneous. Given a polyhomogeneous metric $f$ on $\mathscr{C}$ it is natural to enlarge the atlas on $\mathscr{C} b$ to include all those local coordinate transformations which preserve the polyhomogeneous character of the metric near $\partial \mathscr{l} b$.

We shall say that the initial data $(M, \tilde{g}, \tilde{K})$ are polyhomogeneous if $M$ is as described above and if there exists polyhomogeneous functions $\omega$ and $\omega_{0}$, with $\omega / x \in C^{0}(\bar{M})$, such that the fields $(g, K)$ given by (5.1)-(5.1) are polyhomogeneous. 
We shall say that a development $(V, \tilde{\gamma})$ of polyhomogeneous initial data $(M, \tilde{g}, \tilde{K})$ admits a polyhomogeneous Scri if

1. There exists a manifold with boundary $\bar{V}$ such that $V=\operatorname{int} \bar{V}$, and such that $\partial M$ is a smooth submanifold of $\partial V$;

2. There exist a polyhomogeneous function $\Omega \in C^{1}(\vec{V})$ satisfying $\left.\Omega\right|_{\partial M}=0$, $\nabla \Omega(p) \neq 0$ for $p \in \partial V$, such that the metric $\Omega^{2} \tilde{\gamma}$ is polyhomogeneous;

3. There exists a time function $t$ defined in a neighbourhood of $\bar{M}$ in $\bar{V}$ such that $\left.t\right|_{M}=0$, compatible with the polyhomogeneous structure, with the associated lapse function being uniformly bounded away from zero.

It should be pointed out, that all the results described in Sect. 2 go through under polyhomogeneity conditions on the seed fields $(g, A)$. More precisely, if $g$ is any polyhomogeneous metric on $\bar{M}$ and $A^{i j}$ is any uniformly bounded polyhomogeneous symmetric tensor field on $\bar{M}$, then there exist solutions $(X, \omega)$ of $(2.4)$ and $(2.6)$ such that $L^{\imath j}$ given by (2.5) is polyhomogeneous and uniformly bounded on $\bar{M}$, and $\omega / x$ is polyhomogeneous, uniformly bounded, and uniformly bounded away from zero on $\bar{M}$.

Given a polyhomogeneous initial data set it is natural to ask the question whether or not there exists an obstruction to the existence of a development of the initial data with a polyhomogeneous Scri. We have the following:

Proposition 5.1. Let $(M, \tilde{g}, \tilde{K})$ be a polyhomogeneous initial data set with $g_{i j} \in$ $C^{1}(\bar{M})$ and $K^{i j} \in C^{0}(\bar{M})$. If both for $\varepsilon=1$ and for $\varepsilon=-1$ we have

$$
\left.\left(\lambda_{A B}-\frac{\lambda}{2} h_{A B}\right)\right|_{\partial M_{i}} \neq\left.\varepsilon\left(K_{A B}-\frac{\kappa}{2} h_{A B}\right)\right|_{\partial M_{\imath}}
$$

then there exists no development $(V, \tilde{\gamma})$ of $(M, \tilde{g}, \tilde{K})$ which admits a polyhomogeneous Scri.

Proof. Suppose that a polyhomogeneous Scri exists; therefore one can find a time function $t$ defined in a neighbourhood of $\bar{M}$ in $\bar{V}$ such that $\left.t\right|_{M}=0$, and the associated lapse function $\alpha$ and the shift vector $\beta$ are, in local coordinates, polyhomogeneous, with $\alpha$ uniformly bounded away from zero in a neighbourhood of $\bar{M}$. We have the evolution equations (we use the notation of [19], in particular indices are coordinate indices)

$$
\begin{aligned}
& \frac{\partial g_{i j}}{\partial t}-\mathscr{L}_{\beta} g_{i j}=-2 \alpha K_{i j} \\
& \frac{\partial K_{i j}}{\partial t}-\mathscr{L}_{\beta} K_{i j}=-D_{i} D_{j} \alpha+2 \frac{\alpha}{\Omega}\left(D_{\imath} D_{j} \Omega \nabla_{0} \Omega K_{\imath j}\right) \\
& +\alpha\left[-\frac{3|\nabla \Omega|_{\gamma}^{2}-\Omega \square \Omega}{\Omega^{2}} g_{i j}\right. \\
& \left.+{ }^{3} R_{i j}+K K_{i j}-2 K_{i k} K^{k}{ }_{j}\right] \text {, }
\end{aligned}
$$

where $\alpha$ is the lapse function, $\beta$ is the shift vector, $\mathscr{L}_{\beta}$ denotes a Lie derivative and ${ }^{3} R_{i j}$ is the Ricci tensor of $g_{i j}$. Note that by hypothesis all the functions $\alpha, \beta, g_{i j}$, $\Omega$ and $K^{\imath j}$ are polyhomogeneous and uniformly bounded. If (3.19) does not hold, then the terms $D_{\imath} D_{j} \Omega$ in the trace free part of Eq. (5.8) with $i j=A B$ will give a contribution which blows up as $\Omega^{-1}$ near $\partial M$, while all the remaining terms in the 
trace free part of the $i j=A B$ equation are at most blowing up as some power of $\log x$, which gives a contradiction.

Proposition 5.1 suggests very strongly, that there exists no polyhomogeneous Scri's which are not shear free, even without the extra regularity conditions $g \in C^{1}(\bar{M})$, $K^{i j} \in C^{0}(\bar{M})$ which we have imposed above. Such a result has been observed in [7] in a Bondi-Sachs type setting. Recall that the condition (5.3) has been derived in Sect. 3 precisely under the condition $g \in C^{1}(\bar{M}), K^{i j} \in C^{0}(\bar{M})$. It is rather clear that an analysis of (5.8) under general polyhomogeneity conditions on $(g, K)$ will lead to an analogous "no-go" theorem, with possibly a somewhat modified version of condition (5.6).

Proposition 5.1 shows in particular that generic initial data constructed as in Sect. 2 with smooth seed fields $(g, A)$ will not admit a development which admits a polyhomogeneous Scri (this will hold even when $\omega$ and $L$ are smooth up to boundary as long as (5.6) holds). The above "no-go" result does of course not exclude the possibility of existence of some non-polyhomogeneous conformal completions of $(V, \tilde{\gamma})$.

It may be of some interest to stress that if $V$ is a spacetime which is the maximal development of initial data for which (5.3) holds, then in the class of polyhomogeneous conformal completions one obtains a conformal completion $\bar{V}$ for which $\bar{V} \backslash V$ is a two dimensional smooth manifold, namely $\partial M$. The physical significance of such space-times and completions remains to be investigated.

Consider now a polyhomogeneous initial data set for which (5.5) holds - if this condition were not preserved by evolution via Einstein equations, it would follow from Proposition 5.1 that the resulting space-time could not admit a polyhomogeneous Scri. It is therefore natural to ask the question, whether (5.5) is preserved by evolution. We shall show this is the case for initial data satisfying $g_{i j} \in \not^{p h g} \cap C^{1}(\bar{M})$, $K^{i j} \in \mathscr{A}^{p h g} \cap C^{0}(\bar{M})$, and satisfying moreover the conditions

$$
\tilde{K}=3 \varepsilon, \quad K=0,\left.\quad \lambda_{A}^{A}\right|_{\partial M_{i}}=0 .
$$

It can be shown [7] that for any polyhomogeneous metric Gauss coordinates can be constructed, the coefficients of the transformed metric being again polyhomogeneous in the Gauss coordinate system. In what follows we shall use this coordinate system on $M$ in a neighbourhood of $\partial M_{i}$. Let $n^{i}$ be the three dimensional normal to the sets $\left\{\Omega=c_{1}\right\} \cap\left\{t=c_{2}\right\}$, for some constants $c_{1}, c_{2}$, thus $n^{i}=D^{i} \Omega /|D \Omega|$ and the extrinsic curvature of those sets is given by $\lambda_{A B}=-\mathscr{L}_{n} g_{A B}$, where $\mathscr{L}$ is a Lie derivative. From the evolution equations (5.7)-(5.8) with a little work one finds, assuming (5.5),

$$
\begin{aligned}
\left.\frac{\partial}{\partial t}\left(\lambda_{A B}-\varepsilon K_{A B}\right)\right|_{\partial M_{i}, t=0}= & \varepsilon \mathscr{L}_{\alpha n+\varepsilon \beta}\left(\lambda_{A B}-\varepsilon K_{A B}\right)+\varepsilon \mathscr{D}_{A} \mathscr{D}_{B} \alpha \\
& -\frac{1}{2} \mathscr{L}_{\left[\frac{\partial}{\partial t}-\beta, n\right]} g_{A B}+\kappa g_{A B},
\end{aligned}
$$

with some function $\kappa$. (Fortunately, as explained below, we do not need to use (5.7) - (5.8) to evaluate $\kappa$, which would require quite a messy calculation.) From $\left.K_{i j} n^{j}\right|_{\partial M_{\imath}}=0$ it follows that

$$
\left.\left[\frac{\partial}{\partial t}, n\right]\right|_{\partial M_{\imath}, t=0}=\left.\frac{\partial n}{\partial t}\right|_{\partial M_{\imath}, t=0}=0 .
$$


It should be noted that in (5.9) the lapse function $\alpha$ and the shift vector $\beta$ are arbitrary. We can therefore require that they are polyhomogeneous, and that

$$
\begin{gathered}
\left.\alpha\right|_{\partial M_{\imath}, t=0}=1, \\
\left.n(\alpha)\right|_{\partial M_{\imath}, t=0}=0, \\
\left.(\beta+\varepsilon n)\right|_{\partial M_{i}, t=0}=0 .
\end{gathered}
$$

This leads to

$$
\left.\frac{\partial}{\partial t}\left(\lambda_{A B}-\varepsilon K_{A B}\right)\right|_{\partial M_{\imath}, t=0}=\kappa g_{A B}
$$

(It might be of some interest to point out that with the choice (5.12) we have

$$
\left.\frac{\partial}{\partial t}\right|_{\partial M_{\imath}, t=0}=\left.(\alpha \eta+\beta)\right|_{\partial M_{\imath}, t=0}=-\left.\left(\frac{\varepsilon \alpha}{|D \omega|_{g}} \nabla \Omega\right)\right|_{\partial M_{i}, t=0}
$$

so that at $t=0$ the vector field $\left.\frac{\partial}{\partial t}\right|_{\partial M_{2}, t=0}$ is proportional to $\nabla \Omega$ on $\partial M_{i}$, as should be if we want the integral curves of $\frac{\partial}{\partial t}$ to follow the level set $\Omega=0$.) Consider now the tensor field

$$
\sigma_{A B} \equiv \lambda_{A B}-\frac{1}{2} h^{C D} \lambda_{C D} h_{A B}-\varepsilon\left(K_{A B}-\frac{1}{2} h^{C D} K_{C D} h_{A B}\right)
$$

By definition we have $h^{A B} \sigma_{A B} \equiv 0$, so that

$$
h^{A B} \frac{\partial \sigma_{A B}}{\partial t}=-\frac{\partial h^{A B}}{\partial t} \sigma_{A B} .
$$

Since $\left.\sigma_{A B}\right|_{\partial M_{\imath}, t=0} \equiv 0$ it follows that $\left.h^{A B} \frac{\partial \sigma_{A B}}{\partial t}\right|_{\partial M_{\imath}, t=0}=0$, which together with the definition of $\sigma_{A B}$ and (5.13) yields

$$
\kappa=-\left.\frac{1}{2} \frac{\partial\left(h^{C D} \lambda_{C D}-\varepsilon h^{C D} K_{C D}\right)}{\partial t}\right|_{\partial M_{\imath}, t=0} .
$$

Under the conditions (5.10)-(5.14) that

$$
\left.\frac{\partial \sigma_{A B}}{\partial t}\right|_{\partial M_{\imath}, t=0}=0 .
$$

Equation (5.15) shows that the requirement of preservation of the vanishing of the shear of the conformal boundary doe not lead to any supplementary conditions on the initial data. 


\section{A. Appendix}

For $p \in M$ let $e_{a}=e_{a}{ }^{\mu} \partial_{\mu}, a=1, \ldots, 4$, be an orthonormal tetrad, with

$$
e_{0}^{\nu}=\eta^{\mu}, \quad e_{1}^{\mu}=n^{\mu},
$$

where $\eta^{\mu} \partial_{\mu}$ is a unit timelike vector field orthogonal to $M$ in $(V, \gamma)$, and $n=n^{\mu} \partial_{\mu}$ is any unit vector field tangent to $M$ such that for $p \in \partial M_{i}, n$ is the inward pointing vector orthogonal to $\partial M_{i}$ (such tetrads can easily be constructed by e.g. a GrammSchmidt procedure, with the differentiability class of $e_{i}{ }^{\mu} \partial_{\mu}=e_{i}{ }^{k} \partial_{k}, i, k=1,2,3$, being that of $g_{i j}=\gamma_{i j}$ ). By definition we have

$$
\gamma_{a b}=\gamma_{\mu \nu} e_{a}^{\mu} e_{b}^{\nu}=\operatorname{diag}(-1,1,1,1) .
$$

$\gamma^{a b}=\gamma_{a b} ;$ set $e^{a}=\gamma^{a b} e_{b}$. Define

$$
\omega_{b c}^{a}=\left\langle\nabla_{e_{c}} e_{b}, e^{a}\right\rangle_{\gamma} \Leftrightarrow \nabla_{e_{a}} e_{b}=\omega_{b a}^{c} e_{c}
$$

where $\langle,\rangle_{f}$ is the scalar product in the metric $f$, and $\nabla$ is the Levi-Civita derivative operator of $\gamma$. It follows

$$
\begin{aligned}
\nabla_{a}\left(X^{b} e_{b}\right) & \equiv \nabla_{e_{a}}\left(X^{b} e_{b}\right)=\left(e_{a}\left(X^{b}\right)+\omega_{c a}^{b} X^{c}\right) e_{b} \\
& =:\left(\nabla_{a} X^{b}\right) e_{b} .
\end{aligned}
$$

Let $g$ be the metric induced from $(V, \gamma)$ on $M$, let $h$ be the metric induced from $(M, g)$ on $\partial M_{\imath}$, we shall denote by $D_{i}$, respectively by $\mathscr{D}_{A}$, the Levi-Civita derivative operator of $g$, respectively of $h$. As is well known (cf. e.g. [15]), the tetrad formalism is most convenient in $3+1$ and $2+1$ decomposition calculations because we have

$$
\begin{aligned}
D_{i}\left(X^{j} e_{j}\right) & \equiv D_{e_{\imath}}\left(X^{j} e_{j}\right) \\
& =\left[e_{i}\left(X^{\jmath}\right)+\omega^{j}{ }_{k \imath} X^{k}\right] e_{j} \\
& =:\left(D_{\imath} X^{j}\right) e_{\jmath}, \\
\mathscr{D}_{A}\left(X^{B} e_{B}\right) & \equiv \mathscr{D}_{e_{A}}\left(X^{B} e_{B}\right) \\
& =\left[e_{A}\left(X^{B}\right)+\omega^{B}{ }_{C A} X^{C}\right] e_{B} \\
& =:\left(\mathscr{D}_{A} X^{B}\right) e_{b},
\end{aligned}
$$

where the $\omega$ : . coefficients in (A.3)-(A.4) are the same as those appearing in (A.2). From (A.1) we have

$$
K_{i j}=\omega_{0 i j}=\omega_{0 i j}=\left\langle\nabla_{e_{i}} e_{j}, \eta\right\rangle_{\gamma},
$$

where $K_{i j}$ is the extrinsic curvature tensor of $M$ in $(M, \gamma)$ (the sign conventions here agree with those of [40] when $\eta$ is chosen to be future pointing), and

$$
\begin{aligned}
\lambda_{A B} & =\omega_{1 A B}=\omega_{1 B A}=\left\langle\nabla_{e_{A}} e_{B}, n\right\rangle_{\gamma} \\
& =\left\langle D_{e_{A}} e_{B}, n\right\rangle_{g},
\end{aligned}
$$

where $\lambda_{A B}$ is the extrinsic curvature of $\partial M_{\imath}$ in $(M, g)$ (note the unconventional sign in (A.6) due to the fact that $n$ is the inward pointing normal to $\partial M_{\imath}$. This choice of 
$n$ is convenient for our purposes because if $x$ is a defining function for $\partial M$, then $n=D x /|D x|_{g}$.) The following equations are often used:

$$
\begin{aligned}
\nabla_{i} X_{j} & =D_{i} X_{j}+X_{0} K_{i j}, \\
D_{A} X_{B} & =\mathscr{D}_{A} X_{B}-X_{1} \lambda_{A B}, \\
\nabla_{i} X_{0} & =D_{i} X_{0}+K_{i}{ }^{3} X_{j}, \\
\square \Omega & =-\Omega_{00}+\Delta_{g} \Omega+K \Omega_{0}, \\
\Omega_{00} & \equiv \nabla_{0} \nabla_{0} \Omega, \quad \Omega_{0} \equiv e_{0}(\Omega), \quad K \equiv K_{i}^{i}, \\
\square & \equiv \nabla^{a} \nabla_{a}, \quad \Delta_{g} \equiv D^{i} D_{i} .
\end{aligned}
$$

We have the following embedding equations (cf. e.g. [19])

$$
\begin{aligned}
& R_{\imath j k 0}(\gamma)=D_{j} K_{i k}-D_{i} K_{j k} \\
& R_{i j k l}(\gamma)=R_{\imath \jmath k l}(g)+K_{\imath k} K_{j l}-K_{i l} K_{j k}
\end{aligned}
$$

$R_{a b c d}(f)$ being the Riemann tensor of a metric $f$. We shall need the following well known result:

Proposition A.1. Let $\gamma$ be a conformally vacuum metric on a four dimensional manifold. Then the Weyl tensor $C_{a b c d}$ of $\gamma$ satisfies

$$
C_{i j k l}=2\left(\gamma_{i[k} C_{l] j}+\gamma_{\jmath[l} C_{k] i}\right)
$$

where

$$
C_{i j} \equiv C_{0 i 0 j}
$$

Proof. Since (A.13) is conformally invariant without loss of generality we may assume that $\gamma$ is vaccum. A $3+1$ decomposition calculation gives (cf. e.g. [19])

$$
\begin{gathered}
C_{\imath j}=R_{i \jmath}(g)+\frac{K}{3} L_{\imath j}-L_{i k} L_{j}^{k}+\frac{2}{9} K^{2} g_{i j}, \\
L_{\imath j} \equiv K_{i \jmath}-\frac{K}{3} g_{i j}
\end{gathered}
$$

$\left(g_{\imath \jmath}=\gamma_{i \jmath}\right)$, while from (A.12) one obtains

$$
\begin{gathered}
R_{i j k l}(g)=2 g_{\imath[k} C_{l] \jmath}+2 g_{j[l} C_{k] \imath}+A_{i j k l}, \\
A_{\imath j k l}=2 g_{i[k} L_{l] e} L_{j}^{e}+2 g_{j[l} L_{k] e} L_{i}^{e}+2 L_{i[k} L_{l] j}-|L|_{g}^{2} g_{i[k} g_{l] j}
\end{gathered}
$$

Tracelessness and symmetry of $L_{i j}$ together with the identity

$$
\delta_{[k}^{i} \delta_{l}^{j} L_{e}^{e} L_{f]}^{f}=0
$$

imply $A_{i j k l} \equiv 0$, and the result follows. 


\section{B. Asymptotically Minkowskian Coordinates}

The object of this section is to show that polyhomogeneous initial data sets for which (3.19) holds are asymptotically flat, in the sense that the Cauchy data set can be realised by an embedding of the initial data surface in an asymptotically Minkowskian (not necessarily vacuum) space-time. The notion of asymptotic flatness used here is rather rough, and it is clear from the formulae below that the metric considered possess finer properties than (B.3), we shall however not attempt to formalize that.

Throughout this section, for simplicity of calculations, we shall assume that changing the time orientation if necessary we have

$$
\tilde{K}=-3, \quad K=0 .
$$

(There is little doubt that the results below still hold without these conditions.)

We shall say that $(V, \tilde{\gamma})$ is asymptotically Minkowskian in lightlike directions if there exist constants $R, C_{1}, C_{2}$ and a coordinate system on $V$ covering the set

$$
\mathscr{\odot} \equiv \mathscr{O}_{R, C_{1}, C_{2}} \equiv\left\{(t, x): r \geq R, C_{1}<t-r<C_{2}\right\} \text {, }
$$

and constants $C$ and $N$ such that on 9 we have

$$
\left|\tilde{\gamma}_{\mu \nu}-\eta_{\mu \nu}\right|+\left|\partial_{\sigma} \tilde{\gamma}_{\mu \nu}\right| \leq C r^{-1} \log ^{N} r
$$

We have the following:

Proposition B.1. Let $(M, \tilde{g}, \tilde{K})$ be a polyhomogeneous data set (as defined in Sect. 5) with $g \in C^{1}(M)$ and $K \in C^{0}(\bar{M})$. Suppose moreover that we have $\partial M_{i} \approx S^{2}$, and that (B.1) holds, and that (changing time orientation if necessary) we have

$$
\left.\left(\lambda_{A B}-\frac{h^{C D} \lambda_{C D}}{2} h_{A B}+K_{A B}-\frac{h^{C D} K_{C D}}{2} h_{A B}\right)\right|_{\partial M_{\imath}}=0 .
$$

Then there exists a polyhomogeneous space-time $(V, \tilde{\gamma})$ which is asymptotically Minkowskian in lightlike directions and an embedding $i: M \rightarrow V$ such that $i^{*} \tilde{\gamma}=\tilde{g}$, and $\tilde{K}$ is the extrinsic curvature tensor of $i(M)$ in $(V, \tilde{\gamma})$.

Remarks. 1. We do not claim that $(V, \tilde{\gamma})$ can be chosen vacuum, though we believe that this is indeed the case.

2. The method of proof of Proposition B.1 works without the conditions $g \in C^{1}(\bar{M})$, $K \in C^{0}(\bar{M})$ as long as $g$ and $K$ are polyhomogeneous provided that the coefficients of the $\log ^{i} x$ in terms in $K_{A B}$ are related to some of the coefficients of $x \log ^{i} x$ in $g_{i j}$.

Proof. Let, as usual, $h_{A B}=\left.g_{A B}\right|_{\partial M_{i}}$. As is well known, there exists a conformal diffeomorphism $\Phi: S^{2} \rightarrow S^{2}$ such that $\Phi^{*} h_{A B}=\breve{\phi}^{2} \breve{h}_{A B}$, where $\breve{h}_{A B}$ is the standard round metric on $S^{2}$,

$$
\breve{h}_{A B}=d \theta^{2}+\sin ^{2} \theta d \phi^{2} .
$$

Let us still denote by $g_{i j}$ the metric obtained by pull back with the map $(x, v) \rightarrow$ $(x, \Phi(v))$, we thus have $\left.g_{A B}\right|_{\partial M_{i}}=\breve{\phi}^{2} \breve{h}_{A B}$. Let $\phi$ denote any function on $\bar{M}$ such that $\left.\phi\right|_{\partial M_{i}}=\breve{\phi}$, with $\partial \phi /\left.\partial x\right|_{\partial M_{i}}$ so chosen that the extrinsic curvature of $\partial M_{i}$ in the metric $\phi^{-2} g$ is pure trace. Using once again the symbol $g_{i j}$ for the metric 
$\phi^{-2} g_{\imath j}$ we have thus obtained $\left.g_{A B}\right|_{\partial M_{\imath}}=\breve{h}_{A B}$. As has been shown in [7], for any polyhomogeneous metric Gauss coordinates near the boundary can be introduced, so that we have $g^{x A}=g\left(d x, d x^{A}\right) \equiv 0, g^{x x}=g(d x, d x) \equiv 1$ for $0 \leq x \leq x_{0}$, for some $x_{0}$. We thus have

$$
g_{\imath j} d x^{\imath} d x^{j}=d x^{2}+g_{A B} d x^{A} d x^{B}
$$

with

$$
\left.g_{A B}\right|_{\partial M_{\imath}}=\breve{h}_{A B},\left.\quad \lambda\right|_{\partial M_{\imath}}=-\left.\frac{1}{2} \breve{h}^{A B} \frac{\partial g_{A B}}{\partial x}\right|_{\partial M_{\imath}}=0 .
$$

It now follows from (2.6) that

$$
f \equiv \frac{\omega}{x}=1+O\left(x^{2}\right)
$$

Introducing $r=1 / x$ we have

$$
\tilde{g}_{i \jmath} d x^{\imath} d x^{\jmath}=\omega^{-2} g_{\imath \jmath} d x^{\imath} d x^{\jmath}=f^{-2} r^{-2} d r^{2}+f^{-2} r^{2} g_{A B} d x^{A} d x^{B} .
$$

Define

$$
\tau \equiv t-\sqrt{1+r^{2}}
$$

and set

$$
\tilde{\gamma}_{t t} \equiv-1, \quad \tilde{\gamma}_{t \imath} \equiv 0, \quad \tilde{\gamma}_{i j}(\tau=0)=\chi_{i j}
$$

with

$$
\begin{gathered}
\chi_{r r}=\frac{r^{2}}{1+r^{2}}+\tilde{g}_{r r}=\frac{r^{2}}{1+r^{2}}+\frac{1}{f^{2} r^{2}} \\
\chi_{r A}=0 \\
\chi_{A B}=\tilde{g}_{A B}=\frac{r^{2}}{f^{2}} g_{A B} .
\end{gathered}
$$

It follows that the embedding $\left(r, x^{A}\right) \stackrel{i}{\longrightarrow}\left(t=\sqrt{1+r^{2}}, r, x^{A}\right)$ has the desired property that $i^{*} \tilde{\gamma}=\tilde{g}$, for any $\tilde{\gamma}$ which on $i(M)$ takes the values given by (B.6)-(B.9). To analyze the extrinsic curvature of the slice $\tau=0$ it is useful to pass to the coordinates $\left(\tau, r, x^{A}\right)$ so that the metric

$$
d \tilde{s}^{2}=\tilde{\gamma}_{\mu \nu} d x^{\mu} d x^{\nu}=-d t^{2}+\tilde{\gamma}_{\imath j} d x^{i} d x^{j}
$$

takes the form

$$
d \tilde{s}^{2}=-d \tau^{2}-2 \frac{r}{\sqrt{1+r^{2}}} d \tau d r+\tilde{g}_{i j} d x^{2} d x^{\jmath}
$$

The extrinsic curvature tensor of $i(M)$ will take the desired values if we set

$$
\frac{\partial \tilde{\gamma}_{i \jmath}}{\partial \tau}(\tau=0)=\psi_{i j}
$$


with

$$
\begin{gathered}
\psi_{i j}=-2 \sqrt{\chi_{r r}} f r \tilde{K}_{i j}+\beta^{k} \frac{\partial \tilde{g}_{i j}}{\partial x^{k}}+\frac{\partial \beta^{k}}{\partial x^{2}} \tilde{g}_{k j}+\frac{\partial \beta^{k}}{\partial x^{j}} \tilde{g}_{k i} \\
=2 \sqrt{\chi_{r r}} f r\left[\tilde{g}_{i j}-\frac{r}{f}\left(K_{i j}-\frac{1}{3} g^{k l} K_{k l} g_{\imath j}\right)\right] \\
-\frac{f^{2} r^{3}}{\sqrt{1+r^{2}}} \frac{\partial \tilde{g}_{i \jmath}}{\partial r}+\frac{\partial \beta^{k}}{\partial x^{\imath}} \tilde{g}_{k \jmath}+\frac{\partial \beta^{k}}{\partial x^{j}} \tilde{g}_{k \imath}, \\
\beta^{A}=0, \\
\beta^{r}=-\frac{f^{2} r^{3}}{\sqrt{1+r^{2}}}=-r^{2}+O(1),
\end{gathered}
$$

where $\tilde{g}^{i j}$ is the matrix inverse to $\tilde{g}_{i j}$. One easily finds

$$
\begin{gathered}
\psi_{r r}=2 \sqrt{\chi_{r r}} f r \tilde{g}_{r r}+2 \beta^{r} \frac{\partial \tilde{g}_{r r}}{\partial r}+\frac{\partial \beta^{r}}{\partial r} \tilde{g}_{r r}+O\left(1 / r^{2}\right)=O\left(1 / r^{2}\right) \\
\psi_{r A}=O(1 / r) \\
\psi_{A B}=-2 r^{2}\left(K_{A B}-\frac{1}{2} \frac{\partial h_{A B}}{\partial x}\right)+O\left(r \log ^{N} r\right)=O\left(r \log ^{N} r\right)
\end{gathered}
$$

for some constant $N$ (note that $\psi_{A B}=O\left(r \log ^{N} r\right)$ wouldn't hold if (B.4) weren't satisfied). Let finally $\phi \in C^{\infty}(\mathbb{R})$ be any function satisfying $\operatorname{supp} \phi \subset(-\varepsilon, \varepsilon)$, $0 \leq \phi \leq 1,\left.\phi\right|_{[-\varepsilon / 2, \varepsilon / 2]}=1$, set $\tilde{\phi}(t, r)=\phi\left(t-\sqrt{1+r^{2}}\right)$. Define

$$
\begin{aligned}
\tilde{\gamma}_{\mu \nu} d x^{\mu} d x^{\nu}= & -d t^{2}+(1-\tilde{\phi})\left[d r^{2}+r^{2}\left(d \theta^{2}+\sin ^{2} \theta d \phi^{2}\right)\right] \\
& +\tilde{\phi}\left[\chi_{i \jmath}+\left(t-\sqrt{1+r^{2}}\right) \psi_{i \jmath}\right] d x^{i} d x^{j}
\end{aligned}
$$

It is easily seen that $\varepsilon$ can be chosen small enough so that the metric $\tilde{\gamma}$ satisfies (B.3) in the quasi-Minkowskian cooridnates $(t, x=r \sin \theta \sin \phi, y=r \sin \theta \cos \phi$, $z=r \cos \theta)$ on the set $\left\{r \geq 1 / x_{0}, t \in(-\infty, \infty)\right\}$.

\section{The Extrinsic Curvature of a Null Hypersurface}

Let $(V, \gamma)$ be a Lorentzian manifold, let $\mathscr{N}$ be a null differentiable hypersurface in $V$, i.e., $\mathscr{N}$ is a differentiable submanifold of codimension 1 such that for all $p \in \mathscr{N}$ there exists a neighbourhood $\mathcal{O}_{p}$ of $p$ and a vector field $k$ defined on $\mathscr{O}_{p}$ such that $\forall q \in \mathscr{O}_{p}$, we have $0 \neq k_{q} \in T_{q} \mathscr{N}$ and $\gamma(k, k)=0$. It follows that $k_{q}$ is defined up to the multiplication by a non-zero number. For $X, Y \in T_{p} \mathscr{N}$ define

$$
N(X, Y)=\gamma\left(X, \nabla_{Y} k\right) .
$$

It is easily seen that $N$ is a symmetric tensor field on $\mathscr{N}^{\prime}$ satisfying

$$
\forall X \in T_{p} \mathscr{N}, \quad N(k, X)=0 .
$$

In the space of 2-covariant symmetric tensors let us define the equivalence relation

$$
\begin{gathered}
N_{1} \sim_{1} N_{2} \quad \text { iff } \quad \forall X, Y \in T_{p} \mathscr{N}, \\
N_{1}(X, Y)_{p}=\alpha_{p} N_{1}(X, Y)_{p}, \quad \alpha_{p} \in \mathbb{R} .
\end{gathered}
$$


It follows that the equivalence class $[N]_{1}, N$ given by (C.1), is an object well defined by the geometry of $V$ and by $\mathscr{N} .[N]_{1}$ will be called the extrinsic curvature tensor of $\mathscr{N}$.

Consider next a Lorentzian metric $\gamma_{1}$ on $V$ such that $\gamma_{1}=\phi^{2} \gamma$, where $\phi$ is a differentiable function on $V$; set

$$
X, Y \in T_{p} \mathscr{N}, \quad N_{1}(X, Y)=\gamma_{1}\left(X, \nabla_{Y}^{1} k\right)
$$

where $\nabla^{1}$ is the Levi-Civita covariant derivative of the metric $\gamma_{1}$. One finds

$$
\begin{gathered}
N_{1}(X, Y)=\phi^{2} N(X, Y)+\beta \gamma(X, Y), \\
\beta=-\phi k^{\mu} \partial_{\mu} \phi .
\end{gathered}
$$

In the space of 2-covariant symmetric tensors let us define a second equivalence relation

$$
\begin{gathered}
N_{1} \sim_{2} N_{2} \quad \text { iff } \forall X, Y \in T_{p} \mathscr{N}^{\cdot}, \\
N_{2}(X, Y)_{p}=\alpha_{p} N_{1}(X, Y)_{p}+\beta_{p} \gamma(X, Y), \quad \alpha_{p}, \beta_{p} \in \mathbb{R} .
\end{gathered}
$$

From what has been said it follows that the equivalence class $[N]_{2}, N$ given by (C.1), is an object well defined by the conformal geometry of $V$ and by $\mathscr{N}$. $[N]_{2}$ will be called the conformal extrinsic curvature tensor of $\mathscr{N}$.

\section{Proof of Proposition 3.3}

Consider the equation

$$
S_{a b}=\frac{|D \Omega|_{g}^{2}-\left(\nabla_{0} \Omega\right)^{2}}{\Omega^{2}} \gamma_{a b}-\frac{2 \nabla_{a} \nabla_{b} \Omega}{\Omega}
$$

the equations

$$
\begin{array}{r}
\lim _{M \ni p \rightarrow \partial M_{\imath}}\left[\left(\nabla_{0} \Omega\right)^{2}-|D \Omega|_{g}^{2}\right]=0 \\
\lim _{M \ni p \rightarrow \partial M_{i}}\left[\frac{\left(\nabla_{0} \Omega\right)^{2}-|D \Omega|_{g}^{2}}{\Omega} \gamma_{a b}-2 \nabla_{a} \nabla_{b} \Omega\right]=0,
\end{array}
$$

are clearly necessary conditions for

$$
\left.S_{a b}\right|_{M}=\hat{S}_{a b}+\Omega \log \Omega S_{a b}^{\log },
$$

with $\hat{S}_{a b} \in C^{1}(\bar{M})$, and $S_{a b}^{\log }$ satisfying the 1-condition (we will show that they are also sufficient). Equation (D.2) is easily satisfied by setting

$$
\left.\nabla_{0} \Omega\right|_{M}=\omega_{0},\left.\quad \omega_{0}\right|_{\partial M_{i}}=\left.\varepsilon|D \omega|_{g}\right|_{\partial M_{\imath}}, \quad \varepsilon= \pm 1
$$

$\omega_{0}$ otherwise arbitrary, with an $\varepsilon$ which shall be determined later. A $3+1$ decomposition of the trace-free part of (D.3),

$$
\lim _{M \ni p \rightarrow \partial M_{i}}\left(\nabla_{a} \nabla_{b} \Omega-\frac{1}{4} \square \Omega \gamma_{a b}\right)=0,
$$


gives the equations

$$
\begin{aligned}
\lim _{M \ni p \rightarrow \partial M_{i}} \nabla_{0} \nabla_{0} \Omega & =-\frac{1}{3} \lim _{M \ni p \rightarrow \partial M_{\imath}}\left(\Delta_{g} \omega+K \omega_{0}\right), \\
\lim _{M \ni p \rightarrow \partial M_{i}} D_{i} \omega_{0} & =-\lim _{M \ni p \rightarrow \partial M_{\imath}}\left(K_{i}^{j} D_{j} \omega\right), \\
\lim _{M \ni p \rightarrow \partial M_{i}}\left(D_{i} D_{j} \omega-\frac{1}{3} \Delta_{g} \omega g_{i j}\right) & =-\lim _{M \ni p \rightarrow \partial M_{i}}\left[\omega_{0}\left(K_{i j}-\frac{1}{3} K g_{i j}\right)\right] .
\end{aligned}
$$

Equation (D.6) will be satisfied if we choose

$$
\left.\nabla_{0} \nabla_{0} \Omega\right|_{M}=\omega_{00},\left.\quad \omega_{00}\right|_{\partial M_{\imath}}=-\left.\frac{1}{3}\left(\Delta_{g} \omega+K \varepsilon|D \omega|_{g}\right)\right|_{\partial M_{i}},
$$

$\omega_{00}$ otherwise arbitrary. To analyze (D.7), recall that we have

$$
\tilde{K}^{i j}=\omega^{3} K^{i j}+\omega_{0} \tilde{g}^{i j}
$$

in particular

$$
\tilde{K}=\omega K+\left.3 \omega_{0} \Rightarrow \tilde{K}\right|_{\partial M_{i}}=\left.3 \omega_{0}\right|_{\partial M_{\imath}}
$$

Equation (D.10) gives

$$
\tilde{D}_{i}\left(\tilde{K}^{i j}-\tilde{K} \tilde{g}^{i j}\right)=0 \Leftrightarrow \omega D_{i}\left(K^{i j}-K g^{i j}\right)=2\left(K^{i j} D_{i} \omega+D^{j} \omega_{0}\right),
$$

which shows that (D.7) is automatically satisfied. A $(2+1)$ decomposition of (D.8) with $i=A, j=1$ gives

$$
\left.\mathscr{D}_{A} D_{1} \omega\right|_{\partial M_{i}}=-\left.\left(\omega_{0} K_{1 A}\right)\right|_{\partial M_{\imath}},
$$

where $\mathscr{D}_{A}$ is the Levi-Civita connection of the metric $h_{A B}$ induced on $\partial M_{i}$ by $g$. Equation (D.13) is satisfied because of (D.7) and (D.11). To show that (D.8) with $i=1, j=1$ holds, recall that we have the scalar constraint equation,

$$
\omega \Delta_{g} \omega-\frac{3}{2}|D \omega|_{g}^{2}+\frac{1}{4} \omega^{2}\left(R(g)-|L|_{g}^{2}\right)+\frac{1}{6} \tilde{K}^{2}=0
$$

from which it follows that

$$
\left.|D \omega|_{g}\right|_{\partial M_{i}}=\frac{1}{3}|\tilde{K}|_{\partial M_{i}}
$$

(which is consistent with (D.11)). Applying $e^{1}$ to (D.14) one obtains

$$
\left.\left(2 D_{1} D_{1} \omega+\lambda D_{1} \omega-\frac{1}{3} \tilde{K} D_{1} \tilde{K}\right)\right|_{\partial M_{\imath}}=0
$$

where

$$
\lambda \equiv h^{A B} \lambda_{A B}
$$

with $\lambda_{A B}$ - the extrinsic curvature of $\partial M_{i}$ in $(M, g)$. With a little work from (D.16) one finds that

$$
\left.D_{1} D_{1} \omega\right|_{\partial M_{i}}=-\left.\frac{1}{2}\left[\lambda D_{1} \omega+\left(3 K_{11}-K\right) \omega_{0}\right]\right|_{\partial M_{i}},
$$

which is precisely (D.8) with $i=1, j=1$. It follows that the only non-trivial condition imposed by (D.8) is the equation

$$
\begin{gathered}
{\left.\left[\omega_{0}\left(K_{A B}-\frac{\kappa}{2} h_{A B}\right)\right]\right|_{\partial M_{\imath}}=\left.\left[|D \omega|_{g}\left(\lambda_{A B}-\frac{\lambda}{2} h_{A B}\right)\right]\right|_{\partial M_{i}},} \\
\kappa \equiv h^{A B} K_{A B},
\end{gathered}
$$


which will be satisfied if we choose $\varepsilon$ according to the sign occurring in (3.20). Now the trace of (D.3) gives

$$
\lim _{M \ni p \rightarrow \partial M_{i}}\left(\square \Omega-2 \frac{|D \omega|_{g}^{2}-\left(\omega_{0}\right)^{2}}{\omega}\right)=0,
$$

which with a little effort can be seen to hold if (D.9) holds.

We have thus shown that (D.8) will hold whenever (D.18) holds provided we choose $\omega_{0}$ and $\omega_{00}$ to satisfy (D.5) and (D.9). It is then elementary to show that (D.4) will be satisfied provided $\omega_{0}$ and $\omega_{00}$ are moreover required to satisfy

$$
\begin{aligned}
\omega_{0} & =\hat{\omega}_{0}+\frac{1}{3 !} x^{3} \log x \hat{\omega}_{0}^{\log }, \\
\omega_{00} & =\hat{\omega}_{00}+\frac{1}{2 !} x^{2} \log x \hat{\omega}_{00}^{\log },
\end{aligned}
$$

with $\hat{\omega}_{0} \in C^{3}(\bar{M}), \hat{\omega}_{00} \in C^{2}(\bar{M}), \hat{\omega}_{0}^{\log }$ satisfying the 3-condition, $\hat{\omega}_{00}^{\log }$ satisfying the 2-condition. [This is easily satisfied as we have a complete freedom of choice of the extensions of $\omega$ off $M$. We can e.g. choose $\omega_{0}$ to be any function in $C_{\text {loc }}^{\infty}(M) \cap C^{3}(\bar{M})$ satisfying (D.5), and choose $\omega_{00}$ to be any function in $C_{\text {loc }}^{\infty}(M) \cap C^{2}(\bar{M})$ satisfying (D.9) (with such a choice we will have $\hat{\omega}_{0}^{\log } \equiv 0, \hat{\omega}_{00}^{\log } \equiv 0$; some other choices might be more convenient for some other purposes).] Now the embedding equations (A.11) and (A.12) together with the condition on $g$ and $K$ show that

$$
\begin{aligned}
& \left.R_{i j k 0}\right|_{M}=\hat{R}_{i j k 0}+x \log x \hat{R}_{i j k 0}^{\log }, \\
& \left.R_{i j k l}\right|_{M}=\hat{R}_{i j k l}+x \log x \hat{R}_{i j k l}^{\log },
\end{aligned}
$$

with $\hat{R}_{i j k 0}, \hat{R}_{i j k 0}^{\log }, \hat{R}_{i j k l}, \hat{R}_{i j k l}^{\log }$ satisfying the appropriate conditions. Since

$$
R_{i 0 j 0}=-R_{i j}+g^{k l} R_{i k \jmath l}
$$

Eq. (3.21) follows from (3.23) (which has already been established) and from (D.19)-(D.20).

\section{E. Conventions, Function Spaces}

The summation convention is used throughout this paper. Conventions on signature, covariant derivatives and extrinsic curvatures can be found in Appendix A.

Let $\bar{M}$ be a compact, connected Hausdorff manifold with boundary. Thus $M \equiv$ int $\bar{M}$ is a smooth, paracompact, Hausdorff manifold of dimension 3, and $\partial M \equiv \partial \bar{M}$ is a smooth manifold which is a (necessarily finite) sum of its connected components $\partial M_{i}$. Throughout this paper $x$ will denote a defining function for $\partial M$, i.e. a function satisfying $\left.x\right|_{\partial M}=0, x \geq 0,|d x|_{\partial M}>0$, and the implication $x(p)=0 \Rightarrow p \in \partial M$ holds.

We can always choose a finite number of coordinate charts $\phi_{j}: \mathscr{O}_{j} \rightarrow \mathbb{R}^{n,+} \equiv$ $\left\{y \in \mathbb{R}^{n}: y^{1} \geq 0\right\}, j=1, \ldots, J$, covering a neighbourhood of $\partial M$ such that $y^{1}=x$. When referring to local coordinates we shall implicitly assume that $y^{1}=x$, and we shall use the letter $v$ to denote the coordinate $y^{2}, y^{3}$;

$$
v^{A}=y^{A}, \quad A=2,3 .
$$


Thus

$$
y=(x, v)
$$

The standard Schwartz multi-index notation is used throughout, thus if

$$
\alpha=\left(\alpha_{1}, \ldots, \alpha_{n}\right)
$$

then

$$
\partial^{\alpha}=\partial_{y}^{\alpha}=\partial_{y^{1}}^{\alpha_{1}} \ldots \partial_{y^{n}}^{\alpha_{n}}=\partial_{x}^{\alpha_{1}} \partial_{y^{2}}^{\alpha_{2}} \ldots \partial_{y^{n}}^{\alpha_{n}}=\partial_{x}^{\alpha_{1}} \partial_{v}^{\beta}
$$

where $\beta=\left(\alpha_{2}, \ldots, \alpha_{n}\right)$.

For $k \in \mathbb{N}_{0} \equiv \mathbb{N} \cup\{0\}$ the spaces $C_{\text {loc }}^{k}(M)$ are the spaces of functions $k$ times differentiable on $M$. The spaces $C^{k}(\bar{M})$ are the Banach spaces of functions differentiable $k$-times on $M$ such that $f$ and its derivatives can be extended to continuous functions on $\bar{M}$, and equipped with the supremum norm.

Let $f_{i}$ be a sequence of functions, $f_{i} \in C_{\text {loc }}^{\infty}(M)$, such that for every $N \in \mathbb{N}$ and for all $|\alpha| \leq N$ we have

$$
\left|\partial_{y}^{\alpha} f_{i}\right| \leq C_{i, N} x^{s_{i}, N}
$$

for some sequence $s_{i, N} \underset{\imath \rightarrow \infty}{\longrightarrow} \infty$ and some constants $C_{i, N}$. We shall write

$$
f \sim \sum_{i=0}^{\infty} f_{i}
$$

if for every $n, M \in \mathbb{N}$ there exists $N \in \mathbb{N}$ and a constant $C(n, M)$ such that for all $|\alpha| \leq M$

$$
\left|\partial_{y}^{\alpha}\left(f-\sum_{i=0}^{N} f_{i}\right)\right| \leq C(n, M) x^{n} .
$$

$f$ will be said to be polyhomogeneous, $f \in A_{\text {phg }}$, if $f \in C_{\mathrm{loc}}^{\infty}(M)$ and if there exists a sequence $\left\{N_{j}\right\}_{j=0}^{\infty}, N_{j} \in \mathbb{N}_{0}$, and a sequence of functions $f_{j k} \in C^{\infty}(\bar{M})$ such that

$$
f \sim \sum_{j=0}^{\infty} \sum_{k=0}^{N_{j}} f_{j k} x^{\jmath} \ln ^{k} x .
$$

Let $F$ be a function space over $M$. A tensor field $X=\left(X^{A}{ }_{\beta}\right)$, where $A, \beta$ are some multi-indices, $|A|=r,|\beta|=s$, will be said to belong to $F$ if in local coordinates as described at the beginning of this section the components $X^{A}{ }_{\beta}$ of $X$ are in $F$. We shall write that $X \in x^{\alpha} F$ when $x^{-\alpha} X \in F$, etc. We shall write that $x \in F_{1}+F_{2}$, where $F_{a}, a=1,2$ are two function spaces, if there exist $X_{a} \in F_{a}$ such that $X=X_{1}+X_{2}$.

Acknowledgements. P.T.C. wishes to thank David Singleton and Robert Bartnik for several useful discussions and comments. He also wishes to thank the Department of Mathematics of Université de Tours for friendly hospitality during the final stage of work on this paper. 


\section{References}

1. Andersson, L., Chruściel, P.T.: On the asymptotic behaviour of solutions of the constraint equations in general relativity with "hyperboloidal" boundary conditions. In preparation

2. Andersson, L., Chruściel, P.T., Friedrich, H.: On the regularity of solutions of the Yamabe equation and the existence of smooth hyperbolloidal initial data for Einstein field equations. Commun. Math. Phys. 149, 587-612 (1992)

3. Bondi, H., van der Burg, M.G.J., Metzner, A.W.K.: Proc. Roy. Soc. of London A 269, 21 (1962)

4. Choquet-Bruhat, Y., Christodoulou, D., Francaviglia, M.: Ann. Inst. Henri Poincaré 29, 241 (1978)

5. Choquet-Bruhat, Y., Geroch, R.: Commun. Math. Phys. 14, 329 (1969)

6. Christodoulou, D., Klainerman, S.: The global nonlinear stability of Minkowski space. Princeton Math. Series, Vol. 41, Princeton University Press 1993

7. Chruściel, P.T., MacCallum, M.A.H., Singleton, D.: Gravitational Waves in General Relativity XIV: Bondi Expansions and "Polyhomogeneity" of Scri, gr-qc/9305021, Canberra preprint CMA-MR14-92 (1992)

8. Friedrich, H.: Commun. Math. Phys. 91, 445 (1986)

9. Friedrich, H.: Commun. Math. Phys. 119, 51 (1988)

10. Friedrich, H.: J. Differential Geom. 34, 275 (1993)

11. Friedrich, H., Schmidt, B.: Proc. Roy. Soc. of London A 414, 171 (1987)

12. Geroch, R.: Asymptotic Structure of Space-Time. In: Asymptotic structure of space-time. Esposito, F.P., Witten, L. (eds.) New York, London: Plenum 1977

13. Hawking, S., Ellis, G.F.R.: The large scale structure of space-time. Cambridge: Cambridge University Press 1973

14. Hughes, T., Kato, T., Marsden, J.: Arch. Rat. Mech. Anal. 63, 273 (1976)

15. Misner, C., Thorne, K., Wheeler, J.A.: Gravitation. San Francisco: Freeman 1973

16. Penrose, R., Rindler, W.: Spinors and space-time. Vol. II, Cambridge: Cambridge University Press 1986

17. Penrose, R.: Proc. Roy. Soc. of London A 284, 159 (1965)

18. Sachs, R.K.: Proc. Roy. Soc. of London A 270, 103 (1962)

19. York, J.: Kinematics and Dynamics of general relativity. In: Sources of gravitational radiation. Smarr, L. (ed.) Cambridge: Cambridge University Press 1979

Communicated by S.-T. Yau 